\title{
Atlas of marine bony fish otoliths of Southeastern-Southern Brazil Part VIII: Siluriformes (Ariidae) and Pleuronectiformes (Achiridae, Paralichthyidae, Cynoglossidae)
}

\author{
Cesar Santificetur ${ }^{1}$, Marcella Bockis Giaretta ${ }^{1}$, Valéria Regina Martins Conversani ${ }^{1}$, Marina Rito \\ Brenha-Nunes $^{I}$, Carolina Correia Siliprandi ${ }^{I}$, Carmen Lucia Del Bianco Rossi-Wongtschowski ${ }^{\text {* }}$ \\ ${ }^{1}$ Instituto Oceanográfico da Universidade de São Paulo \\ (Praça do Oceanográfico, 191, 05508-120 São Paulo, SP, Brazil) \\ *Corresponding author: cwongski@usp.br
}

\begin{abstract}
To complement the series of manuscripts published as "Atlas of Teleostei Otoliths of the Southeastern-Southern Brazil", in this volume we present results for species of Siluriformes (two species) and Pleuronectiformes (thirteen species) orders. Considering that the sagittae of Siluriformes are very small we analyzed the left lapillus. Due to the asymmetry of the Pleuronectiformes otoliths, were analysed both left and right structures, being the circumsulcal depression the most important feature to diagnose these species.
\end{abstract}

Descriptors: Sagittae, Lapilli, Morphology, Morphometry, Siluriformes, Pleuronectiformes.

\section{RESUMO}

Em complemento à série de manuscritos publicados como "Atlas de Otólitos para os Teleósteos da região Sudeste-Sul do Brasil", neste volume apresentamos os resultados obtidos para espécies das ordens Siluriformes (duas espécies) e Pleuronectiformes (treze espécies). Tendo em vista que os sagittae dos Siluriformes são muito pequenos, foram analisados os lapilli esquerdos. Devido a assimetria dos Pleuronectiformes, foram analisados tanto sagittae esquerdos quanto direitos, sendo a depressão circumsulcal a feição mais importante para diagnosticar as espécies desta ordem.

Descritores: Sagittae, Lapilli, Morfologia, Morfometria, Siluriformes, Pleuronectiformes. 


\section{INTRODUCTION}

The analysis of otolith shapes is widely applied in taxonomical, phylogenetical, paleontological and food web studies, as well as for the discrimination of populations and other intraspecific forms (FITCH; BROWNELL, 1968; CAMPANA, 2005; \& TUSSET et al, 2008). So, the use of the shape analysis to characterize otolith outlines has become a powerful tool with increasing popularity.

As a contribution for these and other studies, we have been improving the Collection of Teleostei Fish Otoliths of the Southeastern-Southern Brazil (COSS-Brasil) held at the Instituto Oceanográfico - USP, (IOUSP). Nowadays our collection contains around 52,000 pairs of otoliths related to 24 orders, 66 families and 202 species (ROSSIWONGTSCHOWSKI et al., 2016). Also we have been developing a series of papers providing a permanent visual record of otoliths from the Southeastern-Southern Brazilian coast: ROSSI-WONGTSCHOWSKI et al. (2014), SILIPRANDI et al. (2016), BRENHA-NUNES et al. (2016) and two other: SANTIFICETUR et al. 2017 and GIARETTA et al. 2017 GIARETTA et al., 2017 being this volume the last part of the series. Herein, we present the features, measurements and related shape indices of Siluriformes and Pleuronectiformes otoliths.

Usually, lapilli are small, regular-shaped, homogenous otoliths, however use is not common to diagnose most species. With the exception of the Siluriformes order, in which the sagittae are small and extremely fragile, the use of lapilli is more advantageous in the characterization of this group of fish because they are larger (ADAMS, 1940; MARTİNEZ; MONASTERIO DE GONZO, 1991; ASSIS, 2005).

The order Pleuronectiformes includes the flatfishes that are symmetrical and planktonic in the larval phase. Throughout the development, these fish undergo a metamorphosis resulting in asymmetry of the eyes, nerves and muscles of the skull, teeth, scales, paired fins and lateral line. Therefore, due to this asymmetry, the left and right sagittae may be different (CAMPANA, 1984; VOLPEDO; ECHEVERRÍA, 1997; LYCHAKOV et al., 2008) and in that case, it is important to characterize both. Considering that it is not usual to find results about the otolith pair of the flatfish we decided to present them in order to make easy the species diagnosis conducted in different areas especially in Paleontology.

We hope that our papers and our collection will provide a valuable legacy for all sort of studies involving otolith morphology.

\section{MATERIAL AND METHODS}

The characteristics of the study area and the general methodology applied to the sagittae classification are described in ROSSI-WONGTSCHOWSKI et al. (2014), SILIPRANDI et al. (2016), BRENHA-NUNES et al. (2016). Additionally, due to the Pleuronectiformes asymmetry, the circumsulcal depression feature were analyzed and a new classification were proposed (being complete or incomplete) (Figure 1).

We analyzed the left lapillus of the Siluriformes following the terminology of ASSIS (2005), with modifications. The features adopted were: 1) otolith shape, 2) anterior region, 3) posterior region, 4) internal and external edges 5) otolith profile and, two more that are important for these order, 6) suculus lapilli and 7) incisura linea basalis. A new terminology was created for these last features being suculus lapilli: tubular strait and tubular curved (Figure 2) and the incisura linea basalis: absent, shallow, deep and deep bent (Figure 3).

When available three otoliths of each species were illustrated and photographed. The frequency of occurrence of each characteristic was calculated by total length classes (TL), and differences within and among classes have been analyzed applying multiple $\chi^{2}$ test (significance 0.05 ).

The acronyms presented in the shape indices tables are: $\mathrm{T}=$ total fish length, $\mathrm{OL}=$ otolith length, $\mathrm{OH}=$ otolith height and OT $=$ otolith thickness. 
A

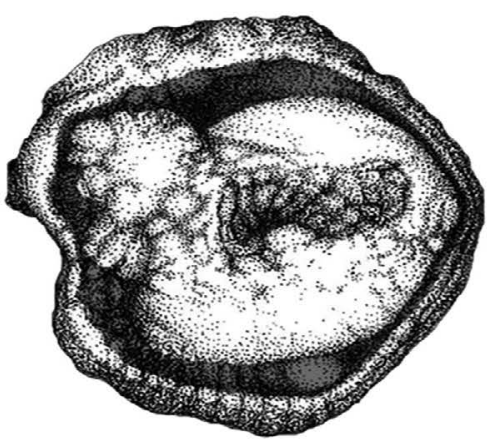

B

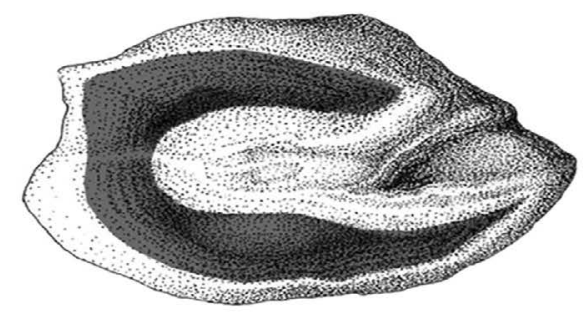

Figure 1. Circumsulcal depression illustrated by the black area: A- Complete (Symphurus tessellatus); B- Incomplete (Paralichthys triocellatus). (Illustration: Alexandre Arackawa).

\section{Sulculus Lapilli}

A

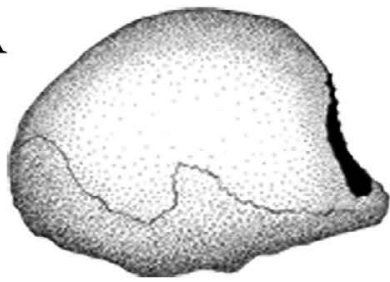

Tubular straight
B

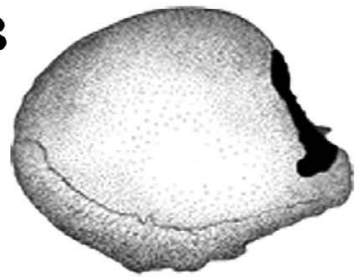

Tubular curved

Figure 2. Proposed terminology for the suculus lapilli (black area). A) Genidens genidens, B) Genidens barbus. (Illustration: Laura Montserrat).

\section{Incisura Linea Basalis}

A

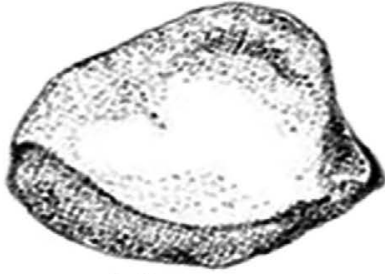

Absent

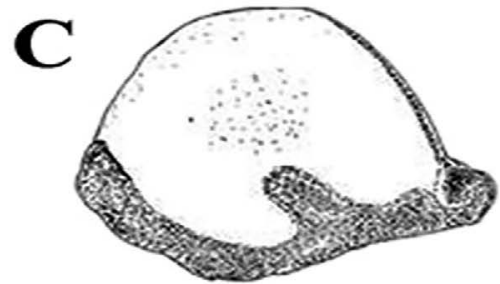

Deep
B

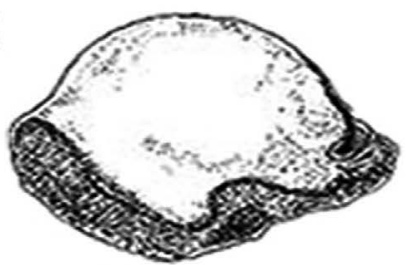

Shallow

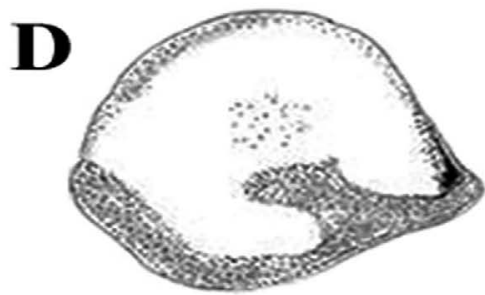

Deep Bent

Figure 3. Proposed terminology for the incisura linea basalis. A) Amphiarius rugispinis, B) Sciades herzbergii, C) and D) Bagreprotocaribbeanus sp. (Adapted from AGUILERA et al., 2013). 


\section{RESULTS}

\section{ORDER SILURIFORMES}

\section{FAMILY ARIIDAE}

\section{Genidens barbus (Lacepède 1803) Plate 1}

$\begin{array}{ll}\begin{array}{ll}\text { Maximum Size: } \\ \text { Distribution: }\end{array} & \begin{array}{l}1200 \mathrm{~mm} \text { (TL) (MARCENIUK; FERRARIS, 2003). } \\ \text { Southwest Atlantic: from La Plata river (Argentina) to eastern of Brazil (FROESE; PAULY, 2016). }\end{array} \\ \text { Habitat: } & \begin{array}{l}\text { Coastal waters and estuaries over the continental shelf; performs anadromous migration during the reproduction } \\ \text { season (VELASCO et al., 2007). }\end{array} \\ \text { Diet: } & \begin{array}{l}\text { Generalist. Feeds on detritus, crustaceans, fish, mollusks and polychaetes (FISCHER et al., 2011). } \\ \text { Colection: }\end{array} \\ \text { Sample: } & 1 \text { otoliths (from } 1 \text { fish (TL 504 mm). }\end{array}$

Shape: oval. Anterior region: oblique-round. Posterior region: round. Lateral edge: entire. Medial edge: entire. Profile: biconvex. Incisura linea basalis: absent. Sulculus lapilli: tubular curved.

Since only one lapillus was analyzed no statistical analysis were done, but its morphometric characteristics are shown below:

\begin{tabular}{lccc}
\hline Shape indices & Mean \pm Sd & Minimum & Maximum \\
\hline OL/TL (\%) & $2.32 \pm 0$ & 2.32 & 2.32 \\
OH/OL (\%) & $95.80 \pm 0$ & 95.80 & 95.80 \\
OT/OL (\%) & $49.53 \pm 0$ & 49.53 & 49.53 \\
OT/OH (\%) & $51.70 \pm 0$ & 51.70 & 51.70 \\
Circularity & $14.07 \pm 0$ & 14.07 & 14.07 \\
Rectangularity & $0.70 \pm 0$ & 0.70 & 0.70 \\
\hline
\end{tabular}

Genidens genidens (Cuvier 1829) Plate 2

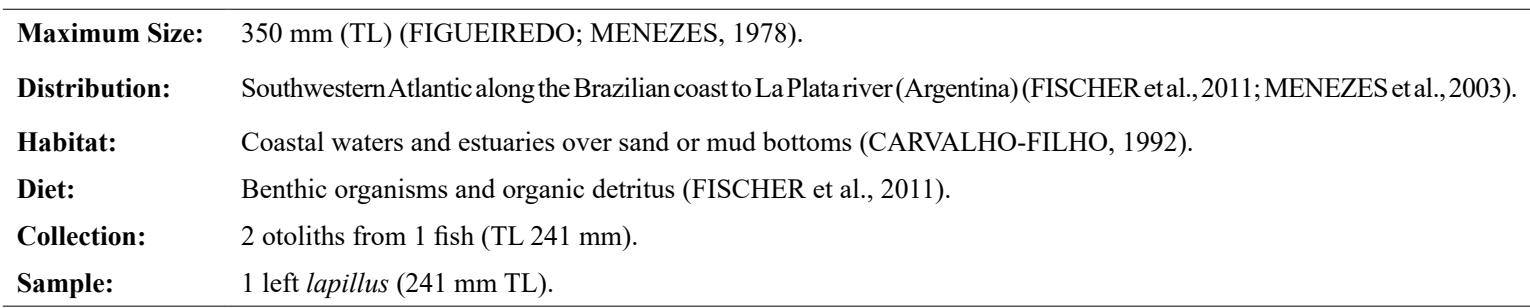

Shape: oval. Anterior region: oblique-round. Posterior region: round. Lateral edge: entire. Medial edge: entire. Profile: biconvex. Incisura linea basalis: deep. Sulculus lapilli: tubular straight.

Since only one lapillus was analyzed no statistical analysis were done but its morphometric characteristics are shown below: 


\begin{tabular}{lccc}
\hline Shape indices & Mean $\pm \mathrm{Sd}$ & Minimum & Maximum \\
\hline OL/TL (\%) & $4.15 \pm 0$ & 4.15 & 4.15 \\
OH/OL (\%) & $92.31 \pm 0$ & 92.31 & 92.31 \\
OT/OL (\%) & $48.55 \pm 0$ & 48.55 & 48.55 \\
OT/OH (\%) & $52.60 \pm 0$ & 52.60 & 52.60 \\
Circularity & $14.26 \pm 0$ & 14.26 & 14.26 \\
Rectangularity & $0.69 \pm 0$ & 0.69 & 0.69 \\
\hline
\end{tabular}

\section{ORDER PLEURONECTIFORMES}

Most of the Pleuronectiformes does not present rostrum and antirostrum so, in this paper these characteristics are described when present.

\section{FAMILY ACHIRIDAE}

Achirus lineatus (Linnaeus 1758) Plates 3 and 4

\begin{tabular}{ll}
\hline Maximum Size: & $230 \mathrm{~mm}$ (TL) (CERVIGÓN et al., 1992). \\
Distribution: & Western Atlantic from South Carolina to northern Argentina (MUNROE, 2002b). \\
Habitat: & Shallow waters and estuaries over sand or mud bottoms to $20 \mathrm{~m}$ deep (FIGUEIREDO; MENEZES, 2000; \\
Diet: & CARVALHO-FILHO, 1992). \\
Collection: & Worms, crustaceans and small fishes (KEITH et al., 2000). \\
Sample: & 1 pair of sagittae (151 mm TL). \\
\hline
\end{tabular}

\section{LEFT OTOLITH}

Shape: bullet-shape. Anterior region: round. Posterior region: flattened. Dorsal edge: sinuate. Ventral edge: sinuate. Profile: concave-convex. Sulcus acusticus: position: median; orientation: horizontal; opening: mesial; morphology: heterosulcoid; colliculum: heteromorphic; ostium: elliptic; cauda: round-oval. Circumsulcal depression: incomplete.

The small number of otoliths did not permit data statistical analysis; however its morphometric characteristics are shown below:

\begin{tabular}{lccc}
\hline Shape indices & Mean \pm Sd & Minimum & Maximum \\
\hline OL/TL (\%) & $2,68 \pm 0$ & 2.68 & 2.68 \\
OH/OL (\%) & $74.75 \pm 0$ & 74.75 & 74.75 \\
OT/OL (\%) & $21.04 \pm 0$ & 21.04 & 21.04 \\
OT/OH (\%) & $28.15 \pm 0$ & 28.15 & 28.15 \\
Circularity & $14.91 \pm 0$ & 14.91 & 14.91 \\
Rectangularity & $0.77 \pm 0$ & 0.77 & 0.77 \\
\hline
\end{tabular}

\section{Right OTOLITH}

Shape: bullet-shape. Anterior region: round. Posterior region: flattened. Dorsal edge: sinuate. Ventral edge: sinuate.

Profile: concave-convex. Sulcus acusticus: position: median; orientation: horizontal; opening: mesial; morphology: heterosulcoid; colliculum: heteromorphic; ostium: elliptic; cauda: round-oval. Circumsulcal depression: incomplete.

The small number of otoliths did not permit data statistical analysis; however its morphometric characteristics are shown below: 


\begin{tabular}{lccc}
\hline Shape indices & Mean \pm Sd & Minimum & Maximum \\
\hline OL/TL (\%) & $2.54 \pm 0$ & 2.54 & 2.54 \\
OH/OL (\%) & $75.72 \pm 0$ & 75.72 & 75.72 \\
OT/OL (\%) & $22.98 \pm 0$ & 22.98 & 22.98 \\
OT/OH (\%) & $30.34 \pm 0$ & 30.34 & 30.34 \\
Circularity & $14.86 \pm 0$ & 14.86 & 14.86 \\
Rectangularity & $0.81 \pm 0$ & 0.81 & 0.81 \\
\hline
\end{tabular}

\section{FAMIILY PARALICHTHYIDAE}

The ostium is frequently elliptic and the cauda morphology is round-oval in most otoliths. The circumsulcal depression is always incomplete and the pseudorostrum and pseudoantirostrum are always absent.

\section{Citharichthys macrops Dresel 1885 Plates 5 and 6}

\begin{tabular}{ll} 
Maximum Size: & $200 \mathrm{~mm}$ (TL) (FROESE; PAULY, 2016). \\
$\begin{array}{l}\text { Distribution: } \\
\text { Habitat: }\end{array}$ & Western Atlantic from North Carolina to southern of Brazil (MENEZES et al., 2003). \\
Diet: & Coastal waters along the continental shelf over hard sand bottom (MUNROE, 2002a). \\
Collection: & 21 otoliths from 13 fish (TL ranging from 35 to $134 \mathrm{~mm}$ ). \\
Sample: & 8 pairs of sagittae categorized into 3, $20 \mathrm{~mm}$ classes (80 to $120 \mathrm{~mm}$ TL). \\
\hline
\end{tabular}

\section{LEFT OTOLITH}

Shape: pentagonal (75\%), bullet-shaped. Anterior region: blunt (63\%), peaked-round. Posterior region: notchedround (50\%), blunt, peaked-round. Anterior dorsal edge: entire (88\%), lobed to sinuate. Dorsal edge: entire (50\%), sinuate, does not apply. Anterior ventral edge: lobed (50\%), entire, sinuate. Posterior ventral edge: sinuate (38\%), lobed, does not apply. Posterior edge: entire (50\%), does not apply, lobed, sinuate. Profile: concave-convex (75\%), plane-convex. Sulcus acusticus: position: median; orientation: descending (63\%), horizontal; opening: mesial; morphology: heterosulcoid; colliculum: heteromorphic; ostium: elliptic (88\%), oval; cauda: round-oval (88\%), elliptic. Circumsulcal depression: incomplete.

The small number of otoliths did not permit data statistical analysis; however its morphometric characteristics are shown below:

\begin{tabular}{lccc}
\hline Shape indices & Mean $\pm \mathrm{Sd}$ & Minimum & Maximum \\
\hline OL/TL (\%) & $2.41 \pm 0.17$ & 2.11 & 2.60 \\
OH/OL (\%) & $79.90 \pm 5.16$ & 72.56 & 87.40 \\
OT/OL (\%) & $17.85 \pm 1.62$ & 15.54 & 20.57 \\
OT/OH (\%) & $22.35 \pm 1.59$ & 20.09 & 25.00 \\
Circularity & $14.66 \pm 0.33$ & 14.24 & 15.28 \\
Rectangularity & $0.73 \pm 0.01$ & 0.70 & 0.74 \\
\hline
\end{tabular}




\section{RIGHT OTOLITH}

Shape: pentagonal (86\%), oval. Anterior region: peaked-round. Posterior region: notched-round (57\%), blunt, flattened, round. Anterior dorsal edge: entire (86\%), sinuate. Dorsal edge: sinuate (43\%), lobed, entire, does not apply. Anterior ventral edge: entire. Posterior ventral edge: entire (71\%), sinuate, does not apply. Posterior edge: entire (71\%), sinuate, does not apply. Profile: concave-convex (86\%), biconvex. Sulcus acusticus: position: median; orientation: descending (86\%), horizontal; opening: mesial; morphology: heterosulcoid; colliculum: heteromorphic; ostium: oval (57\%), elliptic; cauda: elliptic (57\%), round-oval. Circumsulcal depression: incomplete.

The small number of otoliths did not permit data statistical analysis; however its morphometric characteristics are shown below:

\begin{tabular}{lccc}
\hline Shape indices & Mean \pm Sd & Minimum & Maximum \\
\hline OL/TL (\%) & $2.42 \pm 0.14$ & 1.97 & 2.42 \\
OH/OL (\%) & $80.44 \pm 4.99$ & 72.18 & 85.42 \\
OT/OL (\%) & $21.77 \pm 2.41$ & 19.05 & 25.00 \\
OT/OH (\%) & $27.06 \pm 2.51$ & 24.62 & 31.58 \\
Circularity & $14.89 \pm 0.61$ & 13.97 & 15.71 \\
Rectangularity & $0.72 \pm 0.02$ & 0.69 & 0.74 \\
\hline
\end{tabular}

Citharichthys spilopterus Günther 1862 Plates 7 and 8

\begin{tabular}{|c|c|}
\hline Maximum Size: & 200 mm (TL) (ESPÍRITO SANTO et al., 2005). \\
\hline Distribution: & Western Atlantic from New Jersey to Rio Grande do Sul (Brazil) (MENEZES et al., 2003). \\
\hline Habitat: & Coastal waters over mud or sand bottom to $75 \mathrm{~m}$ deep (FISCHER et al., 2011). \\
\hline Diet: & Fishes and small invertebrates (ESPÍRITO SANTO et al., 2005). \\
\hline Collection: & 149 otoliths from 84 fish (TL ranging from 43 to $191 \mathrm{~mm}$ ). \\
\hline Sample: & 37 pairs of sagittae categorized into $7,20 \mathrm{~mm}$ classes ( 40 to $180 \mathrm{~mm} \mathrm{TL}$ ). \\
\hline
\end{tabular}

\section{LEFT OTOLITH}

Shape: pentagonal. Anterior region: peaked. Posterior region: oblique (57\%), blunt, flattened. Anterior dorsal edge: entire. Dorsal edge: entire (97\%), sinuate. Anterior ventral edge: entire. Posterior ventral edge: entire. Posterior edge: entire. Profile: concave-convex. Sulcus acusticus: position: median; orientation: descending (95\%), horizontal; opening: mesial; morphology: pseudo-archaesulcoid; colliculum: heteromorphic; ostium: elliptic; cauda: round-oval. Circumsulcal depression: incomplete.

Statistical differences $(p<0.05)$ within some length classes were obtained for posterior dorsal edge and sulcus acusticus orientation. No differences were found along the fish growth.

\begin{tabular}{lccc}
\hline Shape indices & Mean $\pm \mathrm{Sd}$ & Minimum & Maximum \\
\hline OL/TL (\%) & $2.39 \pm 0.22$ & 1.98 & 2.88 \\
OH/OL (\%) & $77.37 \pm 6.16$ & 64.97 & 89.72 \\
OT/OL (\%) & $18.7 \pm 1.84$ & 15.99 & 23.73 \\
OT/OH (\%) & $24.33 \pm 2.19$ & 20.38 & 31.39 \\
Circularity & $14.54 \pm 0.43$ & 13.62 & 15.55 \\
Rectangularity & $0.72 \pm 0.02$ & 0.67 & 0.77 \\
\hline
\end{tabular}




\section{Right OTOLITH}

Shape: pentagonal. Anterior region: peaked. Posterior region: blunt (80\%), flattened. Anterior dorsal edge: entire. Posterior dorsal edge: entire (94\%), lobed. Anterior ventral edge: entire. Dorsal edge: entire. Posterior edge: entire (94\%), sinuate. Profile: concave-convex. Sulcus acusticus: position: median; orientation: descending; opening: mesial; morphology: pseudo-archaesulcoid; colliculum: heteromorphic; ostium: elliptic; cauda: round-oval. Circumsulcal depression: incomplete.

Statistical differences $(p<0.05)$ within some length classes were obtained for posterior dorsal and posterior edges and posterior region. No differences were found along the fish growth.

\begin{tabular}{lccc}
\hline Shape indices & Mean \pm Sd & Minimum & Maximum \\
\hline OL/TL (\%) & $2.27 \pm 0.22$ & 1.90 & 2.88 \\
OH/OL (\%) & $76.45 \pm 5.82$ & 66.66 & 89.36 \\
OT/OL (\%) & $21.53 \pm 2.95$ & 16.25 & 26.67 \\
OT/OH (\%) & $28.77 \pm 3.97$ & 23.30 & 45.10 \\
Circularity & $14.66 \pm 0.59$ & 13.07 & 16.10 \\
Rectangularity & $0.70 \pm 0.03$ & 0.64 & 0.75 \\
\hline
\end{tabular}

Etropus crossotus Jordan \& Gilbert 1882 Plates 9 and 10

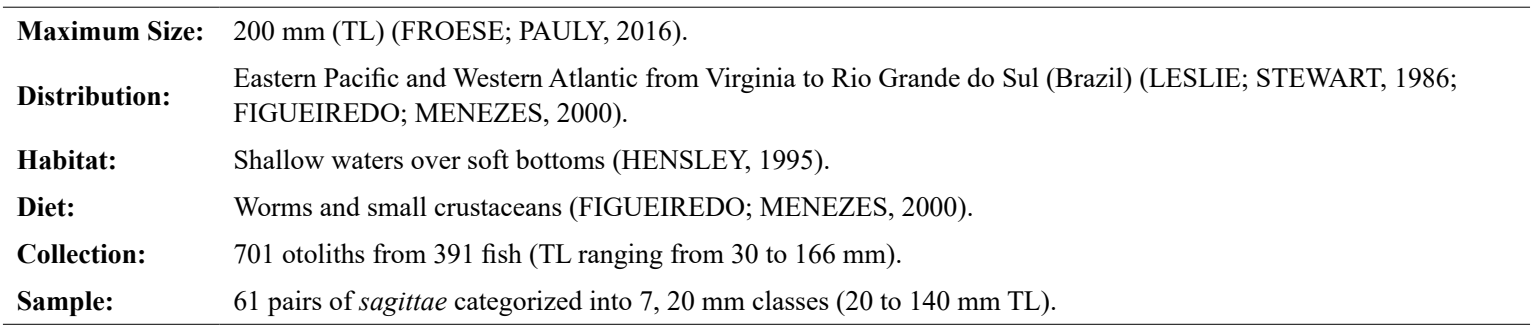

\section{LEFT OTOLITH}

Shape: pentagonal. Anterior region: peaked. Posterior region: oblique (57\%), blunt, flattened. Anterior dorsal edge: entire. Dorsal edge: entire (97\%), sinuate. Anterior ventral edge: entire. Posterior ventral edge: entire. Posterior edge: entire. Profile: concave-convex. Sulcus acusticus: position: median; orientation: descending (95\%), horizontal; opening: mesial; morphology: pseudo-archaesulcoid; colliculum: heteromorphic; ostium: elliptic; cauda: round-oval. Circumsulcal depression: incomplete.

Statistical differences $(p<0.05)$ within some length classes were obtained for shape, anterior and posterior region, posterior ventral, ventral, posterior, dorsal posterior and dorsal edges, sulcus acusticus orientation and morphology, colliculum, ostium and cauda morphology. Along the fish growth statistical differences were found for ventral and posterior dorsal edge.

\begin{tabular}{lccc}
\hline Shape indices & Mean $\pm \mathrm{Sd}$ & Minimum & Maximum \\
\hline OL/TL (\%) & $2.71 \pm 0.33$ & 2.13 & 3.56 \\
OH/OL (\%) & $78.88 \pm 6.17$ & 61.39 & 93.39 \\
OT/OL (\%) & $21.39 \pm 2.63$ & 17.07 & 27.59 \\
OT/OH (\%) & $27.12 \pm 2.45$ & 22.00 & 32.43 \\
Circularity & $14.44 \pm 0.40$ & 13.66 & 15.79 \\
Rectangularity & $0.74 \pm 0.02$ & 0.70 & 0.79 \\
\hline
\end{tabular}




\section{RIGHT OTOLITH}

Shape: pentagonal. Anterior region: peaked. Posterior region: oblique (57\%), blunt, flattened. Anterior dorsal edge: entire. Dorsal edge: entire (97\%), sinuate. Anterior ventral edge: entire. Posterior ventral edge: entire. Posterior edge: entire. Profile: concave-convex. Sulcus acusticus: position: median; orientation: descending (95\%), horizontal; opening: mesial; morphology: pseudo-archaesulcoid; colliculum: heteromorphic; ostium: elliptic; cauda: round-oval. Circumsulcal depression: incomplete.

Statistical differences $(p<0.05)$ within some length classes were obtained for posterior region, posterior ventral and ventral edges, and sulcus acusticus orientation. Along the fish growth statistical differences were found only for ventral edge.

\begin{tabular}{lccc}
\hline Shape indices & Mean \pm Sd & Minimum & Maximum \\
\hline OL/TL (\%) & $2.71 \pm 0.34$ & 2.07 & 3.45 \\
OH/OL (\%) & $77.09 \pm 7.50$ & 59.73 & 93.75 \\
OT/OL (\%) & $23.35 \pm 3.54$ & 17.31 & 33.67 \\
OT/OH (\%) & $30.62 \pm 5.80$ & 21.63 & 43.04 \\
Circularity & $14.74 \pm 0.62$ & 13.76 & 17.18 \\
Rectangularity & $0.70 \pm 0.10$ & 0.65 & 0.80 \\
\hline
\end{tabular}

Etropus longimanus Norman 1933 Plates 11 and 12

\begin{tabular}{ll}
\hline Maximum Size: & $155 \mathrm{~mm}$ (TL) (FIGUEIREDO; MENEZES, 2000). \\
Distribution: & Southwest Atlantic from Rio de Janeiro (Brazil) to Argentina (BERNARDES et al., 2005). \\
Habitat: & Coastal waters to $190 \mathrm{~m}$ deep, commonly found on $40 \mathrm{~m}$ deep (FIGUEIREDO; MENEZES, 2000). \\
Diet: & Polychaetes and small crustaceans (FIGUEIREDO et al., 2002). \\
Collection: & 367 otoliths from 193 fish (TL ranging from 53 to $145 \mathrm{~mm}$ ). \\
Sample: & 44 pairs of sagittae categorized into 6, $20 \mathrm{~mm}$ classes (40 to $140 \mathrm{~mm} \mathrm{TL}$ ). \\
\hline
\end{tabular}

\section{LEFT OTOLITH}

Shape: pentagonal. Anterior region: peaked (70\%), peaked-round. Posterior region: flattened (66\%), blunt, oblique. Anterior dorsal edge: entire. Dorsal edge: entire (84\%), sinuate, lobed to sinuate. Anterior ventral edge: entire (95\%), sinuate. Posterior ventral edge: entire (91\%), sinuate. Posterior edge: entire (95\%), sinuate. Profile: plane-convex. Sulcus acusticus: position: median; orientation: horizontal (77\%), descending; opening: mesial; morphology: archaesulcoid (73\%), pseudo-archaesulcoid; colliculum: homomorphic (73\%), heteromorphic; ostium: absent (73\%), elliptic; cauda: absent (73\%), round-oval. Circumsulcal depression: incomplete.

Statistical differences $(p<0.05)$ within some length classes were obtained for posterior region, posterior ventral, ventral, posterior dorsal and posterior edges, sulcus acusticus orientation and morphology, colliculum, ostium and cauda morphology. Along the fish growth statistical differences were found for sulcus acusticus morphology, colliculum and cauda morphology.

\begin{tabular}{lccc}
\hline Shape indices & Mean \pm Sd & Minimum & Maximum \\
\hline OL/TL (\%) & $2.79 \pm 0.27$ & 2.03 & 3.46 \\
OH/OL (\%) & $84.73 \pm 5.27$ & 73.27 & 93.63 \\
OT/OL (\%) & $23.84 \pm 2.85$ & 18.62 & 31.38 \\
OT/OH (\%) & $28.09 \pm 2.36$ & 23.89 & 34.09 \\
Circularity & $14.06 \pm 0.38$ & 13.40 & 15.09 \\
Rectangularity & $0.73 \pm 0.01$ & 0.70 & 0.76 \\
\hline
\end{tabular}




\section{RIGHT OTOLITH}

Shape: pentagonal (70\%), oval. Anterior region: peaked (82\%), peaked-round. Posterior region: flattened (64\%), round, blunt, oblique. Anterior dorsal edge: entire (95\%), lobed. Dorsal edge: entire (50\%), does not apply, lobed, sinuate. Anterior ventral edge: entire. Posterior ventral edge: entire (66\%), does not apply, sinuate. Posterior edge: entire (59\%), does not apply, sinuate, lobed. Profile: plane-convex. Sulcus acusticus: position: median; orientation: horizontal (70\%), descending; opening: mesial; morphology: archaesulcoid; colliculum: monomorphic; ostium: absent; cauda: absent. Circumsulcal depression: incomplete.

Statistical differences $(p<0.05)$ within some length classes were obtained for shape, anterior and posterior region, posterior ventral, posterior, dorsal and posterior dorsal edges and sulcus acusticus orientation. Along the fish growth statistical differences were found only for sulcus acusticus orientation.

\begin{tabular}{lccc}
\hline Shape indices & Mean $\pm \mathrm{Sd}$ & Minimum & Maximum \\
\hline OL/TL (\%) & $2.77 \pm 0.27$ & 1.99 & 3.42 \\
OH/OL (\%) & $85.24 \pm 5.59$ & 75.47 & 98.20 \\
OT/OL (\%) & $27.06 \pm 3.00$ & 22.05 & 32.35 \\
OT/OH (\%) & $31.74 \pm 2.65$ & 25.26 & 36.17 \\
Circularity & $14.17 \pm 0.43$ & 13.31 & 15.05 \\
Rectangularity & $0.71 \pm 0.02$ & 0.66 & 0.76 \\
\hline
\end{tabular}

Paralichthys isosceles Jordan 1891 Plates 13 and 14

\begin{tabular}{ll}
\hline Maximum Size: & $420 \mathrm{~mm}$ (TL) (BERNARDES et al., 2005). \\
Distribution: & Southwest Atlantic from Rio de Janeiro (Brazil) to northern Patagonia (Argentina) (HAIMOVICI et al., 1994). \\
Habitat: & Demersal species from coastal waters to $190 \mathrm{~m}$ deep (BERNARDES et al., 2005). \\
Diet: & Fishes and cephalopods (BERNARDES et al., 2005). \\
Collection: & 429 otoliths from 219 fish (TL ranging from 197 to $377 \mathrm{~mm}$ ). \\
Sample: & 73 pairs of sagittae categorized into $10,20 \mathrm{~mm}$ classes (180 to $360 \mathrm{~mm} \mathrm{TL})$. \\
\hline
\end{tabular}

\section{LEFT OTOLITH}

Shape: elliptic (75\%), oval. Anterior region: round (82\%), peaked-round, peaked. Posterior region: round. Dorsal edge: sinuate (62\%), entire. Ventral edge: entire (74\%), sinuate. Profile: biconvex (56\%), flattened, plane-convex. Sulcus acusticus: position: median; orientation: horizontal (89\%), ascending; opening: mesial; morphology: heterosulcoid; colliculum: heteromorphic; ostium: tubular; cauda: round-oval. Circumsulcal depression: incomplete.

Statistical differences $(p<0.05)$ within some length classes were obtained for shape, anterior region, ventral and dorsal edges, profile and sulcus acusticus orientation. Along the fish growth statistical differences were found only for profile.

\begin{tabular}{lccc}
\hline Shape indices & Mean $\pm \mathrm{Sd}$ & Minimum & Maximum \\
\hline OL/TL (\%) & $2.43 \pm 0.16$ & 1.94 & 3.19 \\
OH/OL (\%) & $71.11 \pm 5.64$ & 58.98 & 82.79 \\
OT/OL (\%) & $22.72 \pm 2.15$ & 16.93 & 27.44 \\
OT/OH (\%) & $32.12 \pm 3.72$ & 25.74 & 40.68 \\
Circularity & $14.52 \pm 0.5$ & 13.73 & 16.75 \\
Rectangularity & $0.74 \pm 0.02$ & 0.69 & 0.77 \\
\hline
\end{tabular}




\section{RIGHT OTOLITH}

Shape: oval (38\%), elliptic (33\%), rectangular to elliptic (29\%). Anterior region: round (53\%), peaked-round, round. Posterior region: round. Dorsal edge: entire (67\%), sinuate. Ventral edge: entire (86\%), sinuate. Anterior edge: does not apply (71\%), entire. Posterior edge: does not apply (75\%), entire. Profile: pane-convex (67\%), concave-convex. Sulcus acusticus: position: median; orientation: horizontal (60\%), ascending; opening: mesial (92\%), para-ostial, ostial; morphology: heterosulcoid; colliculum: heteromorphic; ostium: elliptic; cauda: round-oval. Circumsulcal depression: incomplete.

Statistical differences $(p<0.05)$ within some length classes were obtained for shape, anterior region, posterior, ventral, dorsal and anterior edges, profile and sulcus acusticus orientation and opening. Along the fish growth statistical differences were found for shape, posterior and dorsal edges and sulcus acusticus orientation.

\begin{tabular}{lccc}
\hline Shape indices & Mean \pm Sd & Minimum & Maximum \\
\hline OL/TL (\%) & $2.34 \pm 0.19$ & 1.74 & 3.32 \\
OH/OL (\%) & $69.76 \pm 5.1$ & 58.45 & 82.25 \\
OT/OL (\%) & $24.30 \pm 3.22$ & 16.88 & 32.73 \\
OT/OH (\%) & $34.96 \pm 4.9$ & 21.95 & 54.11 \\
Circularity & $14.99 \pm 0.80$ & 13.44 & 17.47 \\
Rectangularity & $0.73 \pm 0.04$ & 0.58 & 0.83 \\
\hline
\end{tabular}

Paralichthys patagonicus Jordan 1889 Plates 15 and 16

\begin{tabular}{ll}
\hline Maximum Size: & $570 \mathrm{~mm}$ (TL) (BERNARDES et al., 2005). \\
Distribution: & Western Pacific and Southwest Atlantic from Cabo Frio (Brazil) to Patagonia (Argentina) (FIGUEIREDO; ME- \\
Habitat: & NEZES, 2000; PEQUEÑO; PLAZA, 1987). \\
Diet: & Fissociated to the bottom from coastal waters to $200 \mathrm{~m}$ deep (BERNARDES et al., 2005). \\
Collection: & 268 otoliths from 137 fish (TL ranging from 91 to $555 \mathrm{~mm}$ ). \\
Sample: & 95 pairs of sagittae categorized into $21,20 \mathrm{~mm}$ classes $(80$ to $540 \mathrm{~mm} \mathrm{TL})$. \\
\hline
\end{tabular}

\section{LEFT OTOLITH}

Shape: elliptic (74\%), oval. Anterior region: peaked-round (77\%), peaked, round, double-peaked. Posterior region: round (82\%), flattened. Dorsal edge: sinuate (79\%), lobed, entire. Ventral edge: sinuate (47\%), entire (39\%), lobed, lobed to sinuate. Profile: concave-convex (92\%), biconvex, flattened. Rostrum and antirostrum orientation: does not apply. Rostrum: developed (99\%), absent. Antirostrum: absent (99\%), underdeveloped. Sulcus acusticus: position: median; orientation: horizontal (98\%), ascending; opening: ostial (66\%), para-ostial; morphology: heterosulcoid; colliculum: heteromorphic; ostium: elliptic (93\%), funnel-like; cauda: round-oval. Circumsulcal depression: incomplete.

Statistical differences $(p<0.05)$ within some length classes were obtained for shape, anterior and posterior region, ventral and dorsal edges, profile, sulcus acusticus orientation and opening, rostrum development and ostium morphology. No differences were found along the fish growth.

\begin{tabular}{lccc}
\hline Shape indices & Mean $\pm \mathrm{Sd}$ & Minimum & Maximum \\
\hline OL/TL (\%) & $2.21 \pm 0.15$ & 1.93 & 2.61 \\
OH/OL (\%) & $66.42 \pm 4.68$ & 57.52 & 78.57 \\
OT/OL (\%) & $18.26 \pm 1.67$ & 14.35 & 21.75 \\
OT/OH (\%) & $27.53 \pm 2.1$ & 20.71 & 32.57 \\
Circularity & $16.45 \pm 1.41$ & 14.71 & 22.59 \\
Rectangularity & $0.70 \pm 0.02$ & 0.64 & 0.79 \\
\hline
\end{tabular}




\section{RIGHT OTOLITH}

Shape: elliptic (73\%), oval. Anterior region: peaked-round (77\%), peaked, round, angled. Posterior region: round (88\%), flattened, oblique. Dorsal edge: sinuate (62\%), entire, lobed. Ventral edge: entire (51\%), sinuate, lobed. Profile: concave-convex (98\%), biconvex. Rostrum and antirostrum orientation: does not apply (89\%), in agreement. Rostrum: developed (99\%), absent. Antirostrum: absent (88\%), underdeveloped, developed. Sulcus acusticus: position: median; orientation: horizontal (99\%), ascending; opening: ostial (67\%), para-ostial; morphology: heterosulcoid; colliculum: heteromorphic; ostium: elliptic (92\%), funnel-like; cauda: round-oval. Circumsulcal depression: incomplete.

Statistical differences $(p<0.05)$ within some length classes were obtained for shape, anterior and posterior region, ventral and dorsal edges, profile, sulcus acusticus orientation and opening, rostrum and antirostrum orientation and development and ostium morphology. Along the fish growth statistical differences were found for shape, dorsal edges.

\begin{tabular}{lccc}
\hline Shape indices & Mean \pm Sd & Minimum & Maximum \\
\hline OL/TL (\%) & $2.10 \pm 0.13$ & 1.71 & 2.43 \\
OH/OL (\%) & $65.25 \pm 5.26$ & 47.21 & 93.97 \\
OT/OL (\%) & $21.23 \pm 2.91$ & 14.38 & 27.80 \\
OT/OH (\%) & $32.63 \pm 4.57$ & 22.30 & 47.64 \\
Circularity & $16.33 \pm 1.37$ & 14.39 & 21.37 \\
Rectangularity & $0.70 \pm 0.05$ & 0.63 & 0.98 \\
\hline
\end{tabular}

Paralichthys triocellatus Miranda Ribeiro 1903 Plates 17 and 18

\begin{tabular}{ll}
\hline Maximum Size: & $350 \mathrm{~mm}$ (TL) (BERNARDES et al., 2005). \\
$\begin{array}{l}\text { Distribution: } \\
\text { Habitat: }\end{array}$ & Southwest Atlantic from Cabo Frio, Brazil to Uruguay (FIGUEIREDO; MENEZES, 2000). \\
Diet: & Live between 60 and $192 \mathrm{~m}$ deep (BERNARDES et al., 2005). \\
Collection: & -- \\
Sample: & 248 otoliths from 130 fish (TL ranging from 70 to $338 \mathrm{~mm}$ ). \\
\hline
\end{tabular}

\section{LEFT OTOLITH}

Shape: elliptic (78\%), oval. Anterior region: round (55\%), peaked-round. Posterior region: round (88\%), flattened, angled-round. Dorsal edge: sinuate (80\%), entire. Ventral edge: entire (57\%), sinuate. Profile: biconvex (64\%), concaveconvex, plane-convex. Rostrum and antirostrum orientation: does not apply. Rostrum: absent (75\%), developed, underdeveloped. Antirostrum: absent. Sulcus acusticus: position: median; orientation; horizontal (91\%), ascending; opening: pseudo-ostial (43\%), mesial, para-ostial, ostial; morphology: heterosulcoid; colliculum: heteromorphic; ostium: elliptic (87\%), funnel-like, rectangular; cauda: round-oval. Circumsulcal depression: incomplete.

Statistical differences $(p<0.05)$ within some length classes were obtained for shape, anterior and posterior region, dorsal edges, profile, sulcus acusticus orientation and opening, rostrum development and ostium morphology. Along the fish growth statistical differences were found for anterior region, profile and sulcus acusticus opening.

\begin{tabular}{lccc}
\hline Shape indices & Mean $\pm \mathrm{Sd}$ & Minimum & Maximum \\
\hline OL/TL (\%) & $2.46 \pm 0.24$ & 2.04 & 3.37 \\
OH/OL (\%) & $72.62 \pm 5.77$ & 64.06 & 87.43 \\
OT/OL (\%) & $21.47 \pm 2.68$ & 16.59 & 31.75 \\
OT/OH (\%) & $29.63 \pm 3.42$ & 24.32 & 42.55 \\
Circularity & $14.76 \pm 1.62$ & 13.21 & 26.20 \\
Rectangularity & $0.73 \pm 0.03$ & 0.64 & 0.81 \\
\hline
\end{tabular}




\section{Right OTOLITH}

Shape: oval (68\%), elliptic. Anterior region: round (67\%), peaked, oblique, angled. Posterior region: round. Dorsal edge: entire (80\%), sinuate. Ventral edge: entire (90\%), sinuate, lobed. Profile: biconvex (64\%), concave-convex, planeconvex. Rostrum and antirostrum orientation: does not apply (96\%), in agreement. Rostrum: absent (54\%), developed, underdeveloped. Antirostrum: absent (96\%), developed. Sulcus acusticus: position: median; orientation: horizontal; opening: ostial (67\%), mesial, para-ostial; morphology: heterosulcoid; colliculum: heteromorphic; ostium: elliptic (87\%), funnel-like, rectangular; cauda: round-oval. Circumsulcal depression: incomplete.

Statistical differences $(p<0.05)$ within some length classes were obtained for shape, anterior region, ventral and dorsal edges, profile, sulcus acusticus opening, rostrum and antirostrum development and ostium morphology. Along the fish growth statistical differences were found for shape, anterior region and profile and rostrum development.

\begin{tabular}{lccc}
\hline Shape indices & Mean \pm Sd & Minimum & Maximum \\
\hline OL/TL (\%) & $1.71 \pm 0.28$ & 1.27 & 2.60 \\
OH/OL (\%) & $72.11 \pm 5.59$ & 61.70 & 84.64 \\
OT/OL (\%) & $24.52 \pm 3.7$ & 17.46 & 36.49 \\
OT/OH (\%) & $34.07 \pm 4.95$ & 26.13 & 49.54 \\
Circularity & $20.16 \pm 1.04$ & 12.35 & 14.79 \\
Rectangularity & $0.73 \pm 0.04$ & 0.65 & 0.96 \\
\hline
\end{tabular}

Syacium papillosum (Linnaeus 1758) Plates 19 and 20

\begin{tabular}{ll}
\hline Maximum Size: & $300 \mathrm{~mm}$ (TL) (CARVALHO-FILHO, 1992). \\
Distribution: & Western Atlantic from North Carolina (USA) to Rio Grande do Sul (Brazil) (MUNROE, 2002a). \\
Habitat: & Inhabits shallow waters over sand or mud bottoms to $40 \mathrm{~m}$ deep (CARVALHO-FILHO, 1992). \\
Diet: & Small crustaceans (FIGUEIREDO; MENEZES, 2000). \\
Collection: & 138 otoliths from 71 fish (TL ranging from 60 to $275 \mathrm{~mm})$. \\
Sample: & 44 pairs of sagittae categorized into 9, $20 \mathrm{~mm}$ classes (60 to $260 \mathrm{~mm}$ TL). \\
\hline
\end{tabular}

\section{LEFT OTOLITH}

Shape: bullet-shaped. Anterior region: oblique-round. Posterior region: flattened (60\%), blunt, oblique, round. Dorsal edge: entire (74\%), sinuate, sinuate to entire, lobed to sinuate. Ventral edge: lobed to sinuate (74\%), sinuate to entire, dentate to lobed. Posterior edge: lobed to sinuate (65\%), entire. Profile: concave-convex. Sulcus acusticus: position: median; orientation: ascending; opening: mesial; morphology: heterosulcoid; colliculum: heteromorphic; ostium: elliptic; cauda: straight tubular (98\%), round-oval. Circumsulcal depression: incomplete.

Statistical differences $(p<0.05)$ within some length classes were obtained for posterior region, dorsal, ventral and posterior edges, cauda morphology. No differences were found along the fish growth.

\begin{tabular}{lccc}
\hline Shape indices & Mean \pm Sd & Minimum & Maximum \\
\hline OL/TL (\%) & $2.94 \pm 0.26$ & 2.54 & 4.10 \\
OH/OL (\%) & $76.36 \pm 3.84$ & 66.62 & 83.64 \\
OT/OL (\%) & $22.24 \pm 2.48$ & 16.51 & 27.33 \\
OT/OH (\%) & $29.10 \pm 2.71$ & 23.13 & 33.33 \\
Circularity & $15.60 \pm 1.19$ & 13.85 & 19.16 \\
Rectangularity & $0.79 \pm 0.02$ & 0.75 & 0.83 \\
\hline
\end{tabular}




\section{RIGHT OTOLITH}

Shape: bullet-shaped. Anterior region: oblique-round. Posterior region: flattened (71\%), blunt. Dorsal edge: entire (45\%), lobed to sinuate, lobed, sinuate. Ventral edge: lobed to sinuate (64\%), entire, sinuate, sinuate to entire. Posterior edge: lobed to sinuate (76\%), entire. Profile: concave-convex. Sulcus acusticus: position: median; orientation: ascending; opening: mesial; morphology: heterosulcoid; colliculum: heteromorphic; ostium: elliptic; cauda: straight tubular (98\%), round-oval. Circumsulcal depression: incomplete.

Statistical differences $(p<0.05)$ within some length classes were obtained for posterior region, dorsal, ventral and posterior edges, cauda morphology. Along the fish growth statistical differences were found only for dorsal edge.

\begin{tabular}{lccc}
\hline Shape indices & Mean $\pm \mathrm{Sd}$ & Minimum & Maximum \\
\hline OL/TL (\%) & $2.95 \pm 0.27$ & 2.57 & 4.15 \\
OH/OL (\%) & $77.78 \pm 12.09$ & 67.27 & 89.36 \\
OT/OL (\%) & $21.06 \pm 3.02$ & 14.85 & 28.64 \\
OT/OH (\%) & $26.43 \pm 3.25$ & 20.85 & 35.62 \\
Circularity & $15.45 \pm 1.27$ & 13.78 & 19.64 \\
Rectangularity & $0.75 \pm 0.04$ & 0.68 & 0.82 \\
\hline
\end{tabular}

Xystreurys rasile (Jordan 1891) Plates 21 and 22

\begin{tabular}{ll} 
Maximum Size: & $450 \mathrm{~mm}$ (TL) (CARVALHO-FILHO, 1992). \\
Distribution: & Southwest Atlantic from Rio de Janeiro (Brazil) to Patagonia (Argentina) (MENEZES et al., 2003). \\
Habitat: & Mud and sand bottoms between 5 to $200 \mathrm{~m}$ deep (CARVALHO-FILHO, 1992). \\
Diet: & Amphipods, small crabs and polychaetes (FIGUEIREDO; MENEZES, 2000). \\
Collection: & 310 otoliths from 163 fish (TL ranging from 76 to $392 \mathrm{~mm})$. \\
Sample: & 81 pairs of sagittae categorized into $12,20 \mathrm{~mm}$ classes (60 to $380 \mathrm{~mm}$ TL). \\
\hline
\end{tabular}

\section{LEFT OTOLITH}

Shape: trapezoidal to elliptic (52\%), elliptic (48\%). Anterior region: peaked-round (84\%), peaked, oblique. Posterior region: round (33\%), flattened (32\%), angled (29\%), oblique. Dorsal edge: sinuate (94\%), entire. Ventral edge: entire (51\%), sinuate (49\%). Profile: concave-convex (88\%), plane-convex. Rostrum and antirostrum orientation: does not apply. Rostrum: absent (96\%), developed. Antirostrum: absent. Sulcus acusticus: position: median; orientation: horizontal (93\%), ascending; opening: mesial (93\%), para-ostial, pseudo-ostial; morphology: pseudo-archaesulcoid; colliculum: heteromorphic; ostium: elliptic; cauda: round-oval. Circumsulcal depression: incomplete.

Statistical differences $(p<0.05)$ within some length classes were obtained shape, anterior and posterior region, dorsal, ventral and edges, profile, sulcus acusticus orientation and opening and rostrum development. Along the fish growth statistical differences were found for posterior region, ventral edge and profile.

\begin{tabular}{lccc}
\hline Shape indices & Mean $\pm \mathrm{Sd}$ & Minimum & Maximum \\
\hline OL/TL (\%) & $2.30 \pm 0.16$ & 1.70 & 2.65 \\
OH/OL (\%) & $63.76 \pm 4.40$ & 54.33 & 76.65 \\
OT/OL (\%) & $18.71 \pm 3.64$ & 14.51 & 44.89 \\
OT/OH (\%) & $28.85 \pm 2.65$ & 22.97 & 36.33 \\
Circularity & $15.13 \pm 0.56$ & 14.40 & 17.50 \\
Rectangularity & $0.72 \pm 0.02$ & 0.68 & 0.76 \\
\hline
\end{tabular}




\section{RIGHT OTOLITH}

Shape: elliptic (74\%), trapezoidal to elliptic. Anterior region: peaked-round (89\%), peaked, round. Posterior region: round (54\%), angled, flattened, peaked-round. Dorsal edge: sinuate (75\%), entire. Ventral edge: entire (60\%), sinuate, lobed. Profile: concave-convex (88\%), plane-convex. Rostrum and antirostrum orientation: does not apply. Rostrum: absent. Antirostrum: absent. Sulcus acusticus: position: median; orientation: horizontal (99\%), ascending; opening: mesial (93\%), pseudo-ostial; morphology: pseudo-archaesulcoid; colliculum: heteromorphic; ostium: elliptic; cauda: round-oval. Circumsulcal depression: incomplete.

Statistical differences $(p<0.05)$ within some length classes were obtained shape, anterior and posterior region, dorsal, ventral and edges, profile and sulcus acusticus orientation and opening. Along the fish growth statistical differences were found for ventral edge and profile.

\begin{tabular}{lccc}
\hline Shape indices & Mean $\pm \mathrm{Sd}$ & Minimum & Maximum \\
\hline OL/TL (\%) & $2.31 \pm 0.13$ & 1.89 & 2.63 \\
OH/OL (\%) & $62.86 \pm 4.68$ & 53.17 & 72.98 \\
OT/OL (\%) & $19.05 \pm 2.54$ & 14.71 & 27.33 \\
OT/OH (\%) & $30.32 \pm 3.06$ & 23.94 & 41.36 \\
Circularity & $15.09 \pm 0.59$ & 14.00 & 17.38 \\
Rectangularity & $0.71 \pm 0.02$ & 0.68 & 0.79 \\
\hline
\end{tabular}

\section{FAMILY CYNOGLOSSIDAE}

The otolith shape is frequently discoidal or tall. The anterior region is round and the posterior one flattened. The ostium and cauda are always round-oval. The rostrum, antirostrum, pseudorstrum and pseudoantirostrum are absent and the circumsulcal depression is always complete. The right otolith tends to be larger than the left.

Symphurus tessellatus (Quoy \& Gaimard 1824) Plates 23 and 24

\begin{tabular}{ll}
\hline Maximum Size: & $220 \mathrm{~mm}$ (TL) (FROESE; PAULY, 2016). \\
Distribution: & Western Atlantic from Caribbean Sea to Uruguay (MENEZES et al., 2003). \\
Habitat: & Inhabits waters of the continental shelf over mud bottom at depths between 7 and $110 \mathrm{~m}$ (MUNROE, 2002c). \\
Diet: & Small benthic invertebrates (FIGUEIREDO; MENEZES, 2000). \\
Collection: & 16 otoliths from 9 fish (TL ranging from 103 to $218 \mathrm{~mm}$ ). \\
Sample: & 8 pairs of sagittae categorized into 5, $20 \mathrm{~mm}$ classes (120 to $200 \mathrm{~mm}$ TL). \\
\hline
\end{tabular}

\section{LEFT OTOLITH}

Shape: discoidal. Anterior region: round (75\%), angled-round. Posterior region: round (75\%), flattened. Dorsal edge: entire (88\%), sinuate to entire. Ventral edge: entire (75\%), sinuate to entire. Profile: plane-convex. Sulcus acusticus: position: median; orientation: descending; opening: mesial; morphology: heterosulcoid; colliculum: heteromorphic; ostium: round-oval; cauda: round-oval. Circumsulcal depression: complete.

The small number of otoliths did not permit data statistical analysis; however its morphometric characteristics are shown below:

\begin{tabular}{lccc}
\hline Shape indices & Mean $\pm \mathrm{Sd}$ & Minimum & Maximum \\
\hline OL/TL (\%) & $1.48 \pm 0.09$ & 1.38 & 1.59 \\
OH/OL (\%) & $109.25 \pm 2.81$ & 104.35 & 112.54 \\
OT/OL (\%) & $33.18 \pm 2.60$ & 30.14 & 36.64 \\
OT/OH (\%) & $30.35 \pm 1.92$ & 27.50 & 33.07 \\
Circularity & $13.52 \pm 0.41$ & 13.09 & 14.29 \\
Rectangularity & $0.74 \pm 0.02$ & 0.72 & 0.77 \\
\hline
\end{tabular}




\section{RIGHT OTOLITH}

Shape: discoidal. Anterior region: round. Posterior region: flattened. Dorsal edge: entire (78\%), sinuate. Ventral edge: entire (78\%), sinuate. Profile: plane-convex. Sulcus acusticus: position: median; orientation: horizontal; opening: mesial; morphology: heterosulcoid; colliculum: heteromorphic; ostium: round-oval; cauda: round-oval. Circumsulcal depression: complete.

The small number of otoliths did not permit data statistical analysis; however its morphometric characteristics are shown below:

\begin{tabular}{lccc}
\hline Shape indices & Mean $\pm \mathrm{Sd}$ & Minimum & Maximum \\
\hline OL/TL (\%) & $1.53 \pm 0.11$ & 1.38 & 1.67 \\
OH/OL (\%) & $106.33 \pm 2.64$ & 102.33 & 110.03 \\
OT/OL (\%) & $30.34 \pm 2.32$ & 26.61 & 33.72 \\
OT/OH (\%) & $28.56 \pm 2.44$ & 24.80 & 32.95 \\
Circularity & $13.20 \pm 0.53$ & 11.98 & 14.02 \\
Rectangularity & $0.76 \pm 0.01$ & 0.75 & 0.77 \\
\hline
\end{tabular}

Symphurus jenynsi Evermann \& Kendall 1906 Plates 25 and 26

\begin{tabular}{ll} 
Maximum Size: & $319 \mathrm{~mm}$ (TL) (MUNROE, 1998). \\
Distribution: & Southwest Atlantic from Rio de Janeiro (Brazil) to Argentina (MENEZES et al., 2003). \\
Habitat: & Coastal areas associated to estuaries (MUELBERT; WEISS, 1991). \\
Diet: & Polychaetes and other benthic organisms (MUNROE, 1998). \\
Collection: & 24 otoliths from 13 fish (TL ranging from 138 to $231 \mathrm{~mm}$ ). \\
Sample: & 13 pairs of sagittae categorized into 6,20 mm classes (120 to 220 mm TL). \\
\hline
\end{tabular}

\section{LEFT OTOLITH}

Shape: tall. Anterior region: round. Posterior region: flattened. Dorsal edge: entire. Ventral edge: entire. Profile: plane-convex. Sulcus acusticus: position: median; orientation: horizontal; opening: mesial; morphology: heterosulcoid; colliculum: heteromorphic; ostium: round-oval; cauda: round-oval. Circumsulcal depression: complete.

The small number of otoliths did not permit data statistical analysis; however its morphometric characteristics are shown below:

\begin{tabular}{lccc}
\hline Shape indices & Mean \pm Sd & Minimum & Maximum \\
\hline OL/TL (\%) & $1.31 \pm 0.04$ & 1.26 & 1.36 \\
OH/OL (\%) & $114.13 \pm 5.84$ & 105.36 & 123.83 \\
OT/OL (\%) & $34.86 \pm 5.04$ & 27.59 & 43.95 \\
OT/OH (\%) & $30.57 \pm 4.39$ & 24.10 & 37.50 \\
Circularity & $13.45 \pm 0.25$ & 13.11 & 13.92 \\
Rectangularity & $0.77 \pm 0.02$ & 0.74 & 0.79 \\
\hline
\end{tabular}

\section{Right OTOLITH}

Shape: tall (92\%), discoidal. Anterior region: round. Posterior region: flattened. Dorsal edge: entire. Ventral edge: entire. Profile: biconvex. Sulcus acusticus: position: median; orientation: horizontal; opening: mesial; morphology: heterosulcoid; colliculum: heteromorphic; ostium: round-oval; cauda: round-oval. Circumsulcal depression: complete.

The small number of otoliths did not permit data statistical analysis; however its morphometric characteristics are shown below: 


\begin{tabular}{lccc}
\hline Shape indices & Mean $\pm \mathrm{Sd}$ & Minimum & Maximum \\
\hline OL/TL (\%) & $1.28 \pm 0.05$ & 1.21 & 1.35 \\
OH/OL (\%) & $113.03 \pm 4.70$ & 104.92 & 121.53 \\
OT/OL (\%) & $33.05 \pm 4.84$ & 25.38 & 42.81 \\
OT/OH (\%) & $29.27 \pm 4.34$ & 24.10 & 37.75 \\
Circularity & $13.57 \pm 0.20$ & 13.27 & 13.96 \\
Rectangularity & $0.77 \pm 0.02$ & 0.71 & 0.80 \\
\hline
\end{tabular}

Symphurus plagusia (Bloch \& Schneider 1801) Plates 27 and 28

\begin{tabular}{ll} 
Maximum Size: & $230 \mathrm{~mm}$ (TL) (ESPÍRITO SANTO et al., 2005). \\
$\begin{array}{l}\text { Distribution: } \\
\text { Habitat: }\end{array}$ & Western Atlantic from the Caribbean Sea to Uruguay (ESPÍRITO SANTO et al., 2005). \\
Diet: & Shallow and brackish waters reaching up to $51 \mathrm{~m}$ deep (MUNROE, 2002c) \\
Collection: & Crustaceans and worms (VASCONCELOS FILHO et al., 2010). \\
Sample: & 114 otoliths from 63 fish (TL ranging from 105 to $204 \mathrm{~mm})$. \\
\hline
\end{tabular}

\section{LEFT OTOLITH}

Shape: discoidal. Anterior region: round (96\%), peaked. Posterior region: flattened. Dorsal edge: entire. Ventral edge: entire. Profile: plane-convex. Sulcus acusticus: position: median; orientation: horizontal; opening: mesial; morphology: heterosulcoid; colliculum: heteromorphic; ostium: round-oval; cauda: round-oval. Circumsulcal depression: complete.

Statistical differences $(p<0.05)$ within some length classes were obtained for anterior region. No differences were found along the fish growth.

\begin{tabular}{lccc}
\hline Shape indices & Mean \pm Sd & Minimum & Maximum \\
\hline OL/TL (\%) & $1.52 \pm 0.41$ & 1.25 & 3.31 \\
OH/OL (\%) & $107.19 \pm 4.35$ & 99.13 & 116.09 \\
OT/OL (\%) & $33.33 \pm 4.08$ & 27.14 & 42.86 \\
OT/OH (\%) & $31.08 \pm 3.45$ & 25.11 & 38.92 \\
Circularity & $13.49 \pm 0.31$ & 13.08 & 14.42 \\
Rectangularity & $0.75 \pm 0.02$ & 0.73 & 0.79 \\
\hline
\end{tabular}

\section{Right OTOLITH}

Shape: discoidal (84\%), tall, triangular. Anterior region: round (96\%), peaked. Posterior region: flattened (56\%), round (44\%). Dorsal edge: entire. Ventral edge: entire. Profile: biconvex (96\%), plane-convex. Sulcus acusticus: position: median; orientation: horizontal; opening: mesial; morphology: heterosulcoid; colliculum: heteromorphic; ostium: roundoval; cauda: round-oval. Circumsulcal depression: complete.

Statistical differences $(p<0.05)$ within some length classes were obtained for shape, anterior and posterior regions and profile. No differences were found along the fish growth.

\begin{tabular}{lccc}
\hline Shape indices & Mean \pm Sd & Minimum & Maximum \\
\hline OL/TL (\%) & $1.47 \pm 0.15$ & 1.24 & 1.80 \\
OH/OL (\%) & $105.82 \pm 4.01$ & 98.73 & 113.78 \\
OT/OL (\%) & $31.78 \pm 3.67$ & 26.19 & 40.78 \\
OT/OH (\%) & $30.03 \pm 3.26$ & 25.70 & 37.70 \\
Circularity & $16.73 \pm 16.26$ & 12.97 & 94.77 \\
Rectangularity & $0.75 \pm 0.01$ & 0.73 & 0.79 \\
\hline
\end{tabular}




\section{ACKNOWLEDGMENTS}

We thank: Nicolas Nathan dos Santos for some otoliths measurements and Alexandre Arackawa, Laura Montserrat, Michelle Koing and Vanessa Seiko Sugihara for the otoliths drawings. This paper received substantial financial support from the Fundação de Amparo à Pesquisa do Estado de São Paulo (FAPESP, Process number- 2010/51631-2 and 2014/03764-4).

Special thanks to Dr. Carlos A. Assis for his valuable comments, which greatly improved this paper.

\section{REFERENCES}

ADAMS, L. A. Some characteristic otoliths of American Ostariophysi. J. Morphol., v. 66, n. 3, p. 497-527, 1940.

AGUILERA, O. A; MORAES-SANTOS, H.; COSTA, S.; OHE, F.; JARAMILLO, C.; NOGUEIRA, A. Ariid sea catfish from the coeval Pirabas (Northeastern Brazil), Cantaure, Castillo (Northwestern Venezuela) and Castilletes (North Colombia) formations (Early Miocene), with description of three new species. Swiss J. Palaeontol., v. 132, n. 1, p. 45-68, 2013.

ASSIS, C. A. The utricular otoliths, lapilli, of teleosts: their morphology and relevance for species identification and systematics studies. Sci. Mar., v. 69, n. 2, p. 259-273, 2005.

BERNARDES, R. A.; FIGUEIREDO, J. L.; RODRIGUES, A. R.; FISHER, L. G.; VOOREN, C. M.; HAIMOVICI, M.; ROSSIWONGTSCHOWSKI, C. L. D. B. Peixes da Zona Econômica Exclusiva da região Sudeste-Sul do Brasil: levantamento com armadilhas, pargueiras e rede de arrasto de fundo. São Paulo: EDUSP, 2005. 295 p.

BRENHA-NUNES, M. R.; SANTIFICETUR, C.; CONVERSANI, V. R. M.; GIARETTA, M. B.; ROSSI-WONGTSCHOWSKI, C. L. D. B.; SILIPRANDI, C. C. Atlas of marine bony fish otoliths (sagittae) of Southeastern-Southern Brazil Part IV: Perciformes (Centropomidae, Acropomatidae, Serranidae, Priacanthidae, Malacanthidae, Pomatomidae, Carangidae, Lutjanidae, Gerreidae and Haemulidae). Braz. J. Oceanogr., v. 64, n.spe 1, p. 23-75, 2016.

CAMPANA, S. E. Lunar cycles of otolith growth in the juvenile starry flounder Platichthys stellatus. Mar. BioI., v, 80, n. 3, p. $239-246,1984$.

CAMPANA, S. E. Otolith science enrering the 21st century. Marine and Freshwater Research, 56, 485-495, 2005.

CARVALHO FILHO, A. Peixes: costa brasileira. São Paulo, Marca D’Água. 304 p. 1992.

CERVIGÓN, F.; CIPRIANI, R.; FISCHER, W.; GARIBALDI, L.; HENDRICKX, M.; LEMUS, A. J.; MÁRQUEZ, R.; POUTIERS, J. M.; ROBAINA, G.; RODRIGUEZ, B. Fichas FAO de identificación de especies para los fines de la pesca: guía de campo de las especies comerciales marinas y de aquas salobres de la costa septentrional de Sur América. Rome: FAO, 1992. 513 p.

ESPÍRITO SANTO, R.V.; ISSAC, V. J.; SILVA, L. M. A.; MARTINELLI, J. M.; HIGUCHI, H.; SAINT-PAUL, U. Peixes e camarões do estuário do litoral de bragantino, Pará, Brasil. Belém: MADAM, 2005. 268 p.

FIGUEIREDO, J. L.; MENEZES, N. A. Manual de peixes marinhos do Sudeste do Brasil. II. Teleostei (1). São Paulo: Museu de Zoologia da Universidade de São Paulo, 1978. 110 p.

FIGUEIREDO, J. L.; MENEZES, N. A. Manual de peixes marinhos do Sudeste do Brasil. VI. Teleostei (5). São Paulo: Museu de Zoologia da Universidade de São Paulo, 2000. 116 p.

FIGUEIREDO, J. L.; SANTOS, A. P.; YAMAGUTI, N.; BERNARDES, R. A.; ROSSI-WONGTSCHOWSKI, C. L. D. B. Peixes da zona econômica exclusiva da região sudeste-sul do Brasil: Levantamento com rede de meia-água. São Paulo: EDUSP, 2002. 242 p.

FISCHER, L. G.; PEREIRA, L. E. D.; VIEIRA, J. P. Peixes estuarinos e costeiros. Série Biodiversidade do Atlântico Sudoeste. Rio Grande: Ecoscientia, 2011. 46 p.

FITCH, J.E., BROWNELL, R. L. Fish otolith in cetacean stomachs and their Importance in interpreting feeding habits. J.Fish. Res. Bd. Canada, 25(12): 2561-2574, 1968.

FROESE, R.; PAULY. D. FishBase. World Wide Web electronic publication. Available: <http://www.fishbase.org>. Version: $01 / 2016$.

GIARETTA, M. B.; SILIPRANDI, C. C.; SANTIFICETUR, C.; BRENHA-NUNES, M. R.; CONVERSANI, V. R. M.; ROSSIWONGTSCHOWSKI, C. L. D. B. Atlas of marine bony fish otolith (sagittae) of Southeastern-Southern Brazil Part VI: Albuliformes, Anguiliformes, Osmeriformes, Stomiiformes, Aulopiformes, Myctophiformes, Ophidiiformes, Polimixiiformes, Batrachoidiformes and Lophiformes. Braz. J. Oceanogr, 65(2):278-308. 2017.

HAIMOVICI, M.; MARTINS, A. S.; FIGUEIREDO, J. L.; VIEIRA, P. C. Demersal bony fish of the outer shelf and upper slope of the southern Brazil Subtropical Convergence Ecosystem. Mar. Ecol. Prog. Ser., v. 108, p. 59-77, 1994.

HENSLEY, D. A. Paralichthyidae. Lenguados. In: FISCHER, W.; KRUPP, F.; SCHNEIDER, W.; SOMMER, C.; CARPENTER, K. E.; NIEM, V. (Eds.). Guia FAO para Identification de Especies para lo Fines de la Pesca. Pacifico Centro-Oriental. 3 Vols. Rome: FAO, 1995. p. 1349-1380.

KEITH, P.; LE BAIL, P. Y.; PLANQUETTE, P. Atlas des poissons d'eau douce de Guyane. Tome 2, Fascicule I: Batrachoidiformes, Mugiliformes, Beloniformes, Cyprinodontiformes, Synbranchiformes, Perciformes, Pleuronectiformes, Tetraodontiformes. Collection Patrimoines Naturels. Paris: Publications scientifiques du Muséum national d'Histoire naturelle, 2000. 286 p.

LESLIE JR, A. J.; STEWART, D. J. Systematics and Distributional Ecology of Etropus (Pisces, Bothidae) on the Atlantic Coast of the United States with Description of a New Species. Copeia, v. 1986, n. 1, p. 140-156, 1986.

LYCHAKOV, D. V.; REBANE, Y. T.; LOMBARTE, A.; DEMESTRE, M.; FUIMAN, L. A. Saccular otolith mass asymmetry in adult flatfishes. J. Fish Biol., v. 72, n. 10, p. 2579-2594, 2008 
MARCENIUK, A. P.; FERRARIS JR, C. J. Ariidae (Sea catfishes). In: REIS, R. E.; KULLANDER S. O.; FERRARIS JR, C. J. (Eds.). Checklist of the freshwater fishes of South and Central America. Porto Alegre: Edipucrs, 2003. p. 447-455.

MARTINEZ, V.; MONASTERIO DE GONZO, G. Clave de identificación de algunos peces Siluriformes en base al estudio de sus otolitos. Rev. Asoc. Cienc. Nat. Litoral, v. 2, n. 22, p. 95-118, 1991.

MENEZES, N. A.; BUCKUP, P. A.; FIGUEIREDO, J. L.; MOURA, R. L. (Eds.). Catálogo das espécies de peixes marinhos do Brasil. São Paulo: Museu de Zoologia da Universidade de São Paulo, 2003. 160 p.

MUELBERT, J. H.; WEISS, G. Abundance and distribution of fish larvae in the channel area of the Patos Lagoon Estuary, Brazil. In: HORTY, R. D. (Ed.). Larval fish recruitment and research in the Americas. Proceedings of the $13^{\text {th }}$ Annual Fish Conference. Merida, México. NOAA Technical Report NMFS, 1991. p. 43-54.

MUNROE, T. A. Systematics and ecology of tonguefishes of the genus Symphurus (Cynoglossidae: Pleuronectiformes) from the western Atlantic Ocean. Fish. Bull., v. 96, n. 1, p. 1-182, 1998.

MUNROE, T. A. Paralichthyidae. In: CARPENTER, K. E. (Ed.). The living marine resources of the Western Central Atlantic. Bony fishes part 2 (Opistognathidae to Molidae), sea turtles and marine mammals. FAO Species Identification Guide for Fishery Purposes and American Society of Ichthyologists and Herpetologists Special Publication No. 5. Rome: FAO, 2002a. p. 1375-2127.

MUNROE, T. A. Achiriidae. In: CARPENTER, K. E. (Ed.) The living marine resources of the Western Central Atlantic. Bony fishes part 2 (Opistognathidae to Molidae), sea turtles and marine mammals. FAO Species Identification Guide for Fishery Purposes and American Society of Ichthyologists and Herpetologists Special Publication No. 5. Rome, FAO. 2002b. p. 1375-2127.

MUNROE, T. A. Cynoglossidae. In: CARPENTER, K. E. (Ed.) The living marine resources of the Western Central Atlantic. Bony fishes part 2 (Opistognathidae to Molidae), sea turtles and marine mammals. FAO Species Identification Guide for Fishery Purposes and American Society of Ichthyologists and Herpetologists Special Publication No. 5. Rome, FAO. 2002c. p. 1375-2127.

PEQUEÑO, G.; PLAZA, R. Descripcion de Paralichthys delfini n. sp., con notas sobre otros lenguados congenericos de Chile (Pleuronectiformes, Bothidae). Rev. Biol. Mar., v. 23, n. 2, p. 159-172, 1987.

ROSSI-WONGTSCHOWSKI, C. L. D. B.; CHALOM, A.; SILIPRANDI, C. C.; BRENHA-NUNES, M. R.; CONVERSANI, V. R. M.; SANTIFICETUR, C.; GIARETTA, M. B. COSS-Brasil: Coleção de Otólitos de Peixes Marinhos da Região Sudeste-Sul do Brasil. São Paulo: Instituto Oceanográfico da Universidade de São Paulo, 2016.

ROSSI-WONGTSCHOWSKI, C. L. D. B.; SILIPRANDI, C. C.; BRENHA, M. R.; GONSALES, S. A.; SANTIFICETUR. C.; VAZ-DOSSANTOS, A. M. Atlas of marine bony fish otoliths (Sagittae) of Southeastern - Southern Brazil Part I: Gadiformes (Macrouridae, Moridae, Bregmacerotidae, Phycidae and Merlucciidae); Part II: Perciformes (Carangidae, Sciaenidae, Scombridae and Serranidae). Braz. J. Oceanogr., v. 62, n.spe. 1, p. 1-103, 2014.

SANTIFICETUR, C.; CONVERSANI, V. R. M.; BRENHA-NUNES, R. B GIARETTA, M. B.; SILIPRANDI, C. C.; ROSSIWONGTSCHOWSKI, C. L. D. B. Atlas of marine bony fish otoliths (sagittae) of Southeastern-Southern Brazil Part V: Perciformes (Sparidae, Scianidae, Polynemidae, Mullidae, Kyphosidae, Chaetodontidae, Mugilidae, Scaridae, Percophidae, Pinguipedidae, Blenniidae Gobiidae, Ephippidae, Sphyraenidae, Gempylidae, Trichiuridae, Scombridae, Ariommatidae, Stromateidae and Caproidae). Braz. J. Oceanogr, 65(2):201-257. 2017.

SILIPRANDI, C. C.; BRENHA-NUNES, M. R.; ROSSI-WONGTSCHOWSKI, C. L. D. B.; SANTIFICETUR, C. Atlas of marine bony fish otoliths (sagittae) of Southeastern-Southern Brazil Part III: Clupeiformes (Clupeidae, Engraulidae, Pristigasteridae). Braz. J. Oceanogr., v. 64, n.spe. 1, p. 1-22, 2016.

TUSET, V. M.; LOMBARTE, A., ASSIS, C. A. Otolith atlas for the western Mediterranean, north and central eastern Atlantic. Scientia Marina 72(S1): 7-198, 2008

VASCONCELOS FILHO, A. L.; NEUMANN-LEITÃO, S.; ESKINAZI-LEÇA, E.; OLIVEIRA, A. M. E. Hábitos alimentares de peixes consumidores secundários do Canal de Santa Cruz, Pernambuco, Brasil. Trop. Oceanogr. Recife, v. 38, n. 2, p. 122-129, 2010.

VELASCO, G.; REIS, E. G.; VIEIRA, J. P. Calculating growth parameters of Genidens barbus (Siluriformes, Ariidae) using length composition and age data. J. Appl. Ichthyol., v. 23, n. 1, p. 64-69, 2007.

VOLPEDO A. V.; ECHEVERRÍA D. D. Morfología de la sagitta de lenguados del Mar Argentino (Pisces: Pleuronectiformes). Thalassas, v. 13, p. 113-126, 1997. 

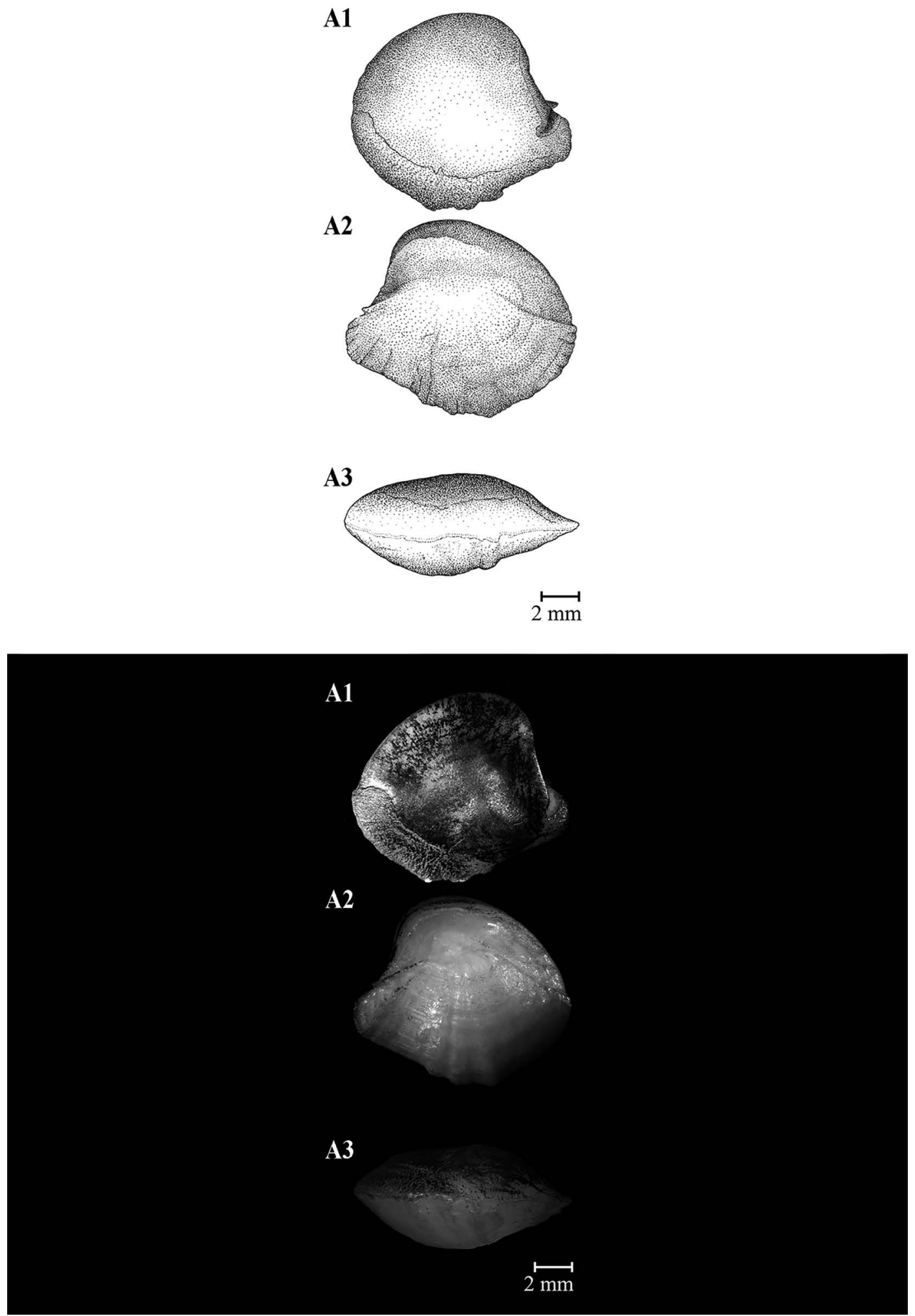

Plate 1. Illustrations (above) and photographs (below) of Genidens barbus lapillus from fish with total length: A. $504 \mathrm{~mm}$. The ventral face is shown in A1; the dorsal face in A2; and the medial face in A3; (Illustration: Laura Montserrat; Photos: Cesar Santificetur). 

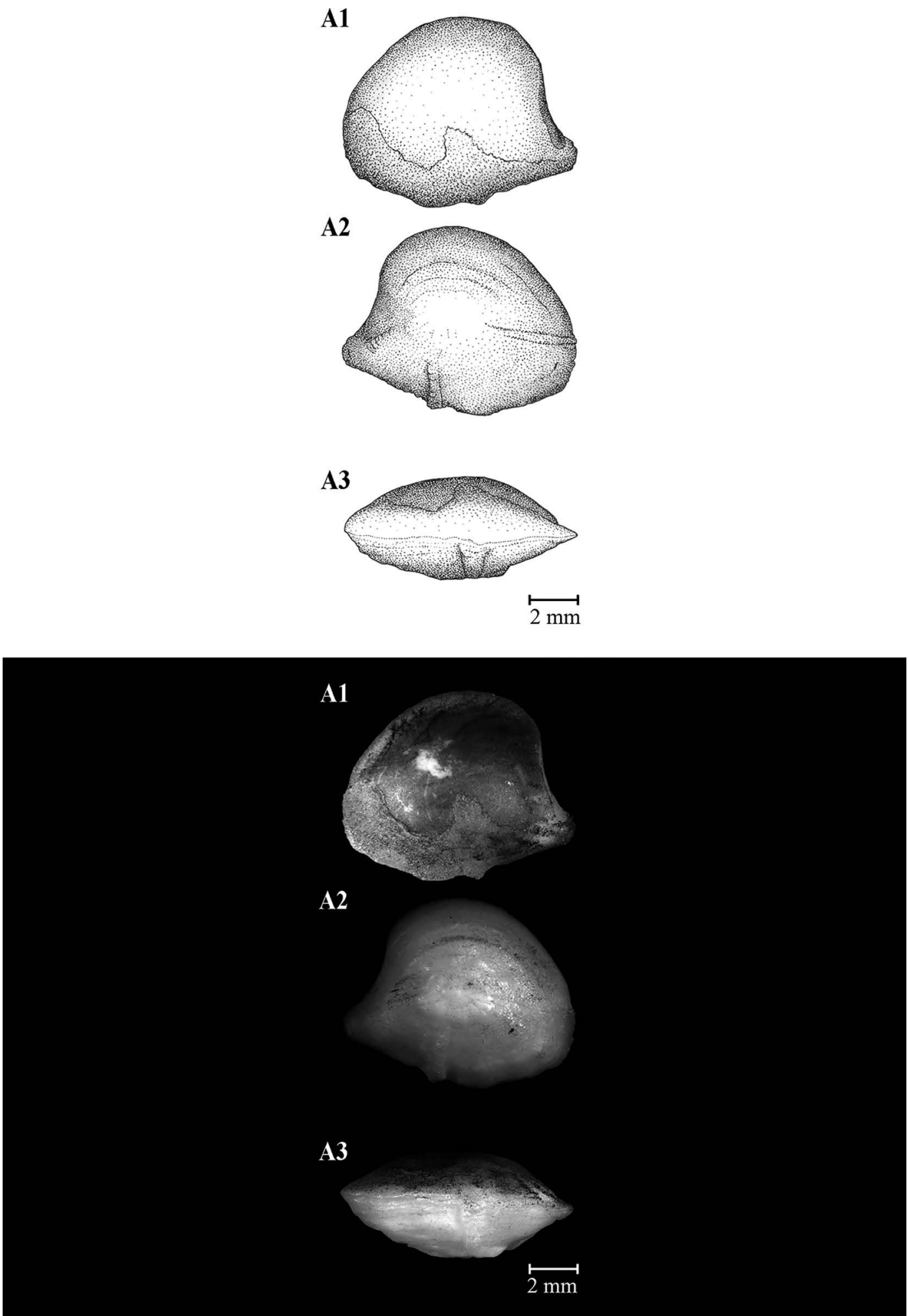

Plate 2. Illustrations (above) and photographs (below) of Genidens genidens lapillus from fish with total length: A. $241 \mathrm{~mm}$. The ventral face is shown in A1; the dorsal face in A2; and the medial face in A3 (Illustration: Laura Montserrat; Photos: Cesar Santificetur). 


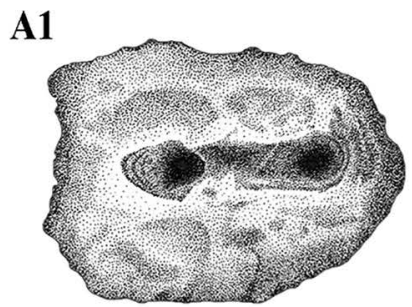

A2

A3
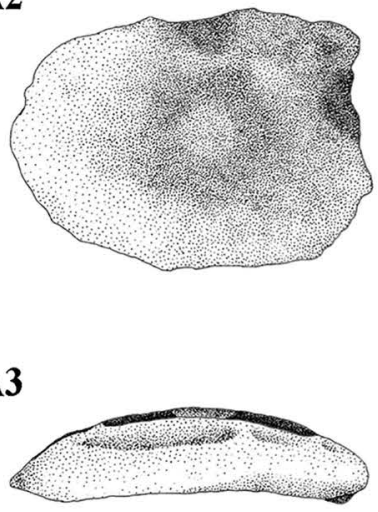

$1 \mathrm{~mm}$

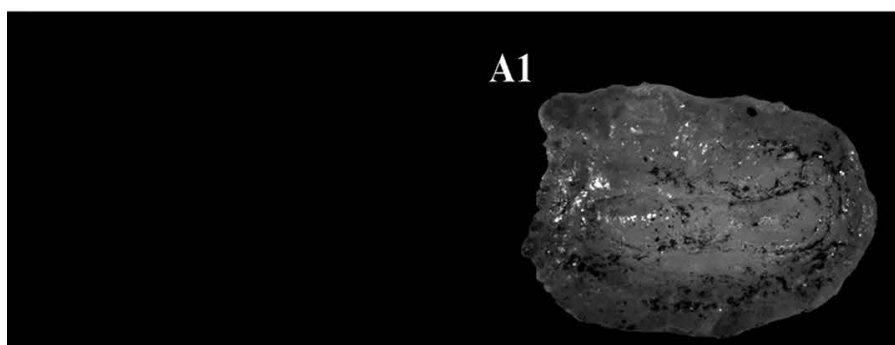

A2

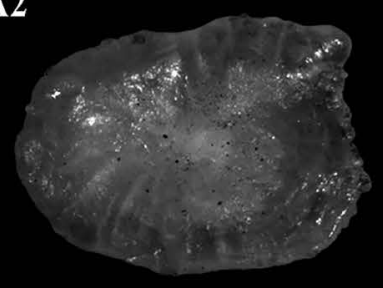

A3

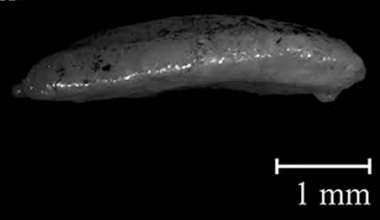

Plate 3. Illustrations (above) and photographs (below) of Achirus lineatus left sagitta from fish with total length: A. $151 \mathrm{~mm}$. The medial face is shown in A1; the lateral face in A2; and the ventral profile in A3 (Illustration: Vanessa Seiko Sugihara; Photos: Cesar Santificetur). 
A1

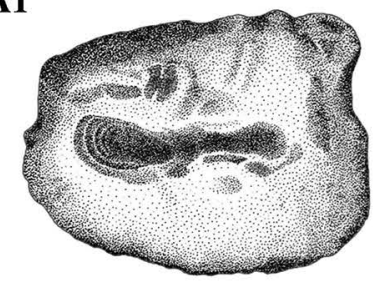

A2

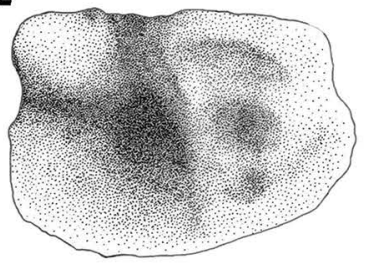

A3

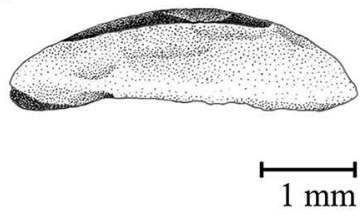

A1

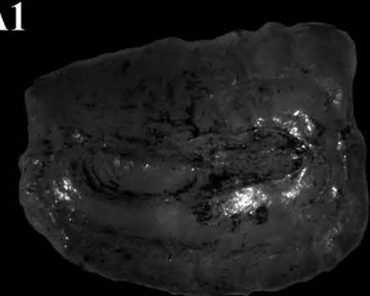

$\mathbf{A 2}$

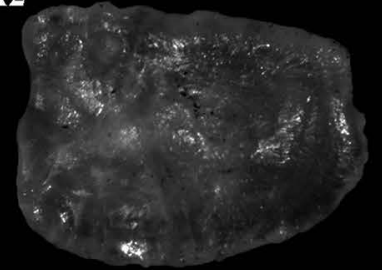

A3

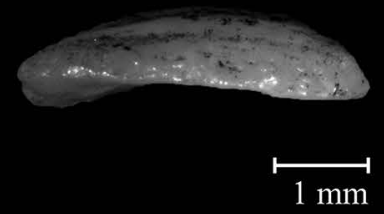

Plate 4. Illustrations (above) and photographs (below) of Achirus lineatus right sagitta from fish with total length: A. $151 \mathrm{~mm}$. The medial face is shown in A1; the lateral face in A2; and the ventral profile in A3 (Illustration: Vanessa Seiko Sugihara; Photos: Cesar Santificetur). 


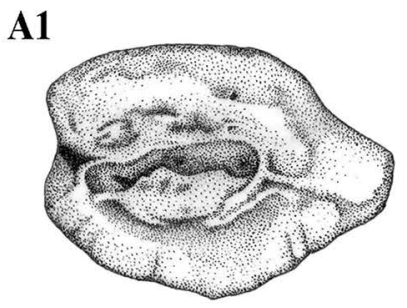

\section{A2}

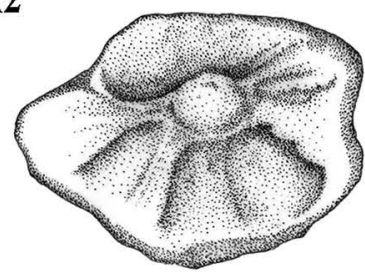

A3

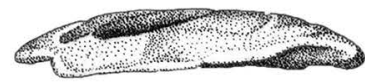

$1 \mathrm{~mm}$
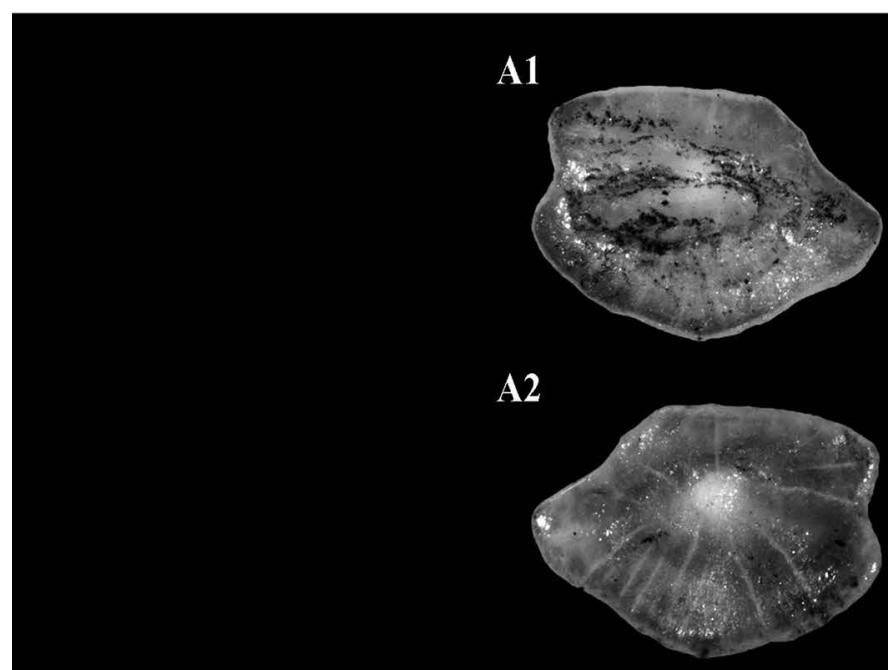

A3

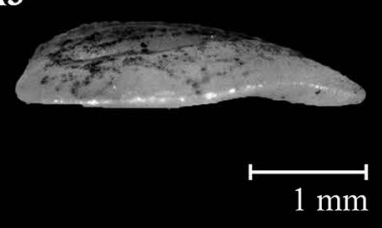

Plate 5. Illustrations (above) and photographs (below) of Citharichthys macrops left sagitta from fish with total length: A. $134 \mathrm{~mm}$. The medial face is shown in A1; the lateral face in A2; and the ventral profile in A3 (Illustration: Michelle Konig; Photos: Cesar Santificetur). 


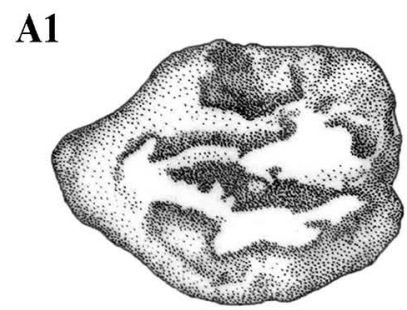

\section{A2}

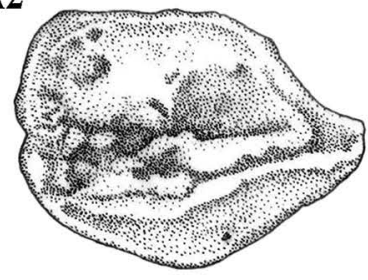

A3

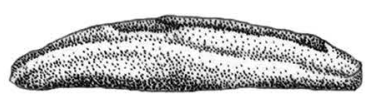

$1 \mathrm{~mm}$

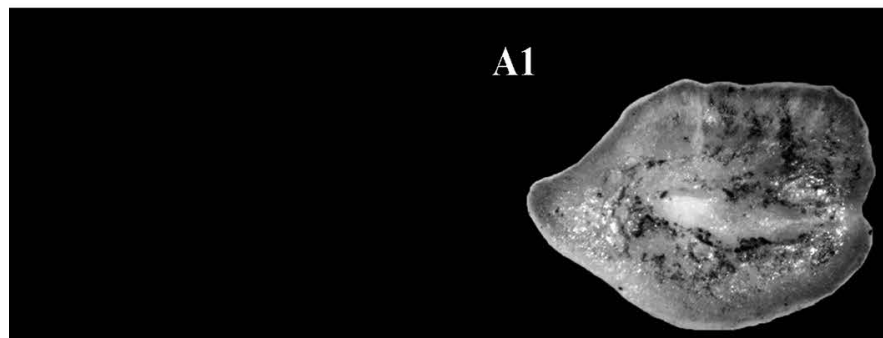

$\mathbf{A 2}$

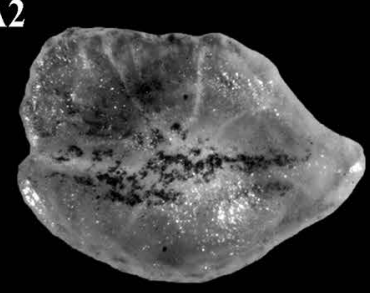

$\mathbf{A 3}$

Plate 6. Illustrations (above) and photographs (below) of Citharichthys macrops right sagitta from fish with total length: A. $134 \mathrm{~mm}$. The medial face is shown in A1; the lateral face in A2; and the ventral profile in A3 (Illustration: Michelle Konig; Photos: Cesar Santificetur). 
A1

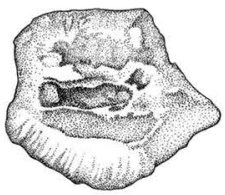

A2

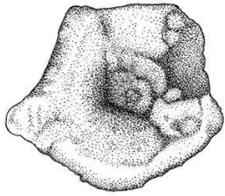

A3

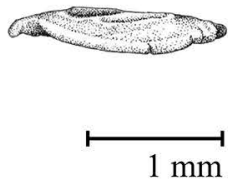

B1

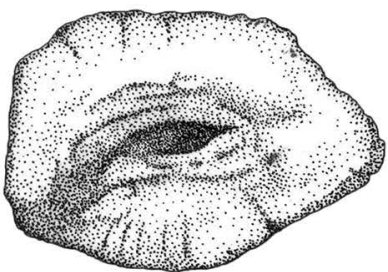

B2

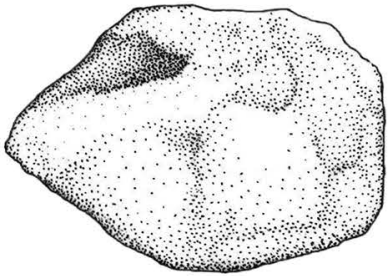

B3
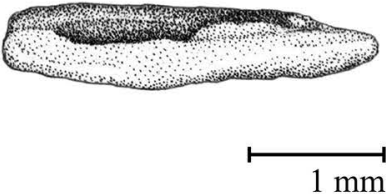

A1

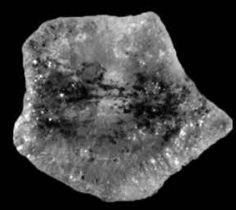

A2

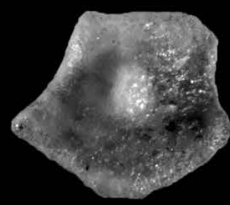

A3

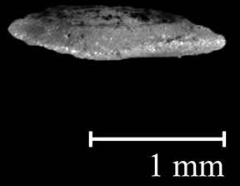

B1

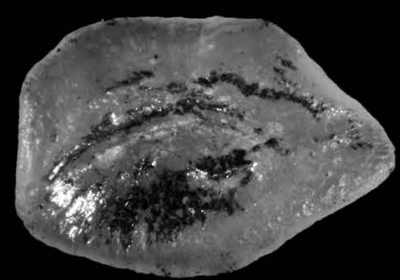

B2

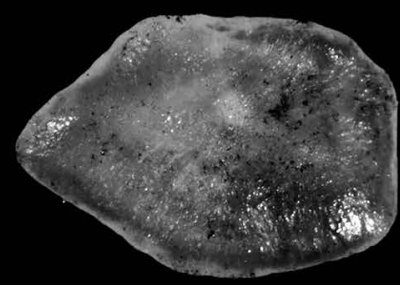

B3

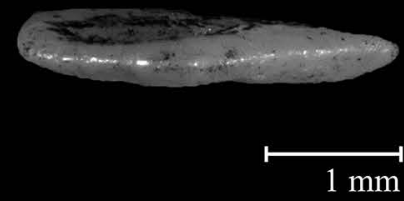

Plate 7. Illustrations (above) and photographs (below) of Citharichthys spilopterus left sagittae from fish with total length: A. 63 mm; B. 183 $\mathrm{mm}$. The medial face is shown in A1; B1; the lateral face in A2; B2; and the ventral profile in A3; B3 (Illustration: Michelle Konig; Photos: Cesar Santificetur). 
A1

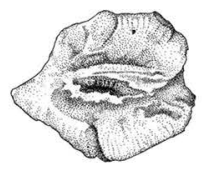

A2

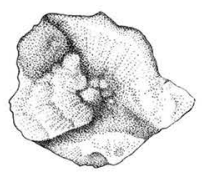

A3

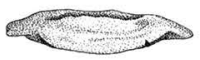

$1 \mathrm{~mm}$
B1

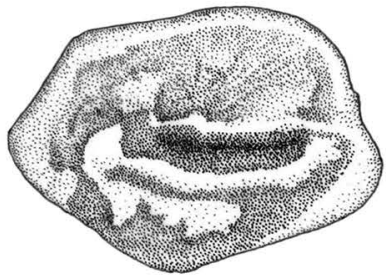

B2

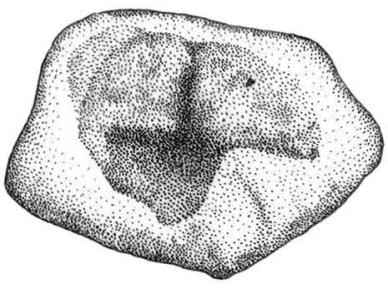

B3

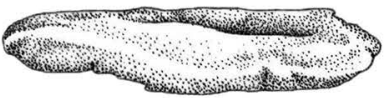

$1 \mathrm{~mm}$
A1

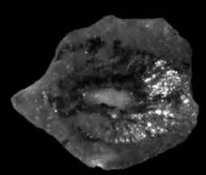

A2

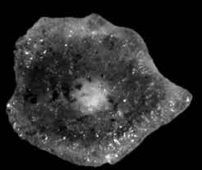

A3

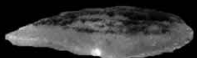

$\longmapsto$
B1

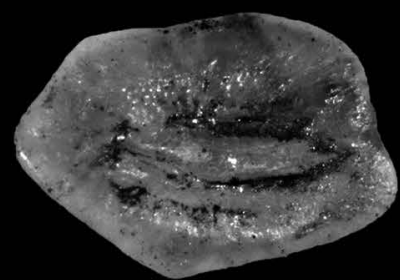

B2

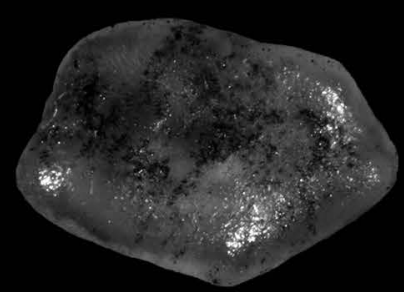

B3

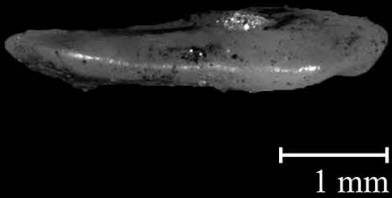

Plate 8. Illustrations (above) and photographs (below) of Citharichthys spilopterus right sagittae from fish with total length: A. 63 mm; B. 183 $\mathrm{mm}$. The medial face is shown in A1; B1; the lateral face in A2; B2; and the ventral profile in A3; B3 (Illustration: Michelle Konig; Photos: Cesar Santificetur). 
A1

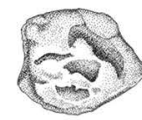

A2

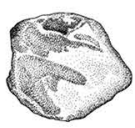

A3
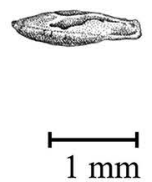

B1

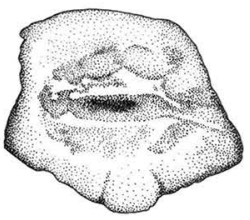

B2

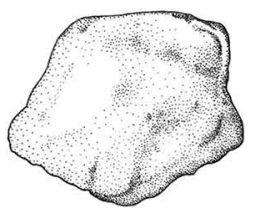

B3
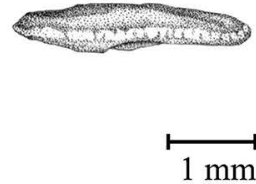

C1

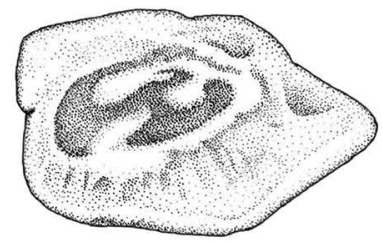

$\mathrm{C} 2$

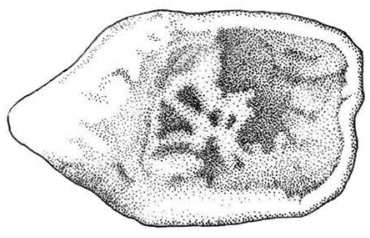

C3

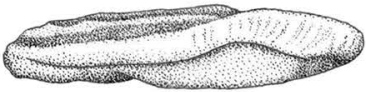

A1

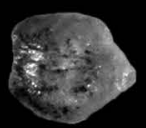

A2

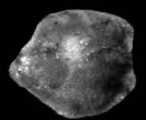

$\mathbf{A 3}$
B1

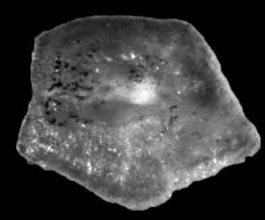

B2

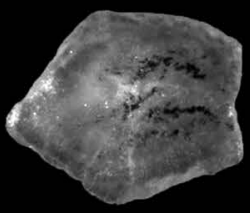

B3

\section{C1}

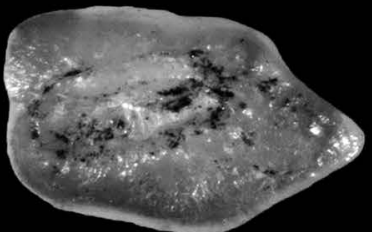

C2

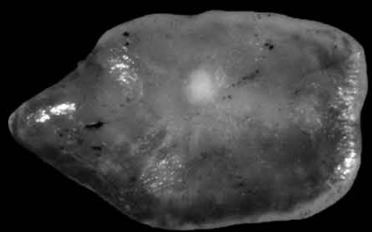

C3

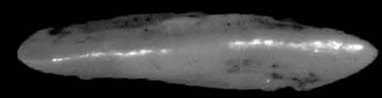

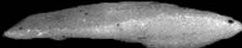

$\stackrel{\mathrm{mm}}{\longmapsto}$

Plate 9. Illustrations (above) and photographs (below) of Etropus crossotus left sagittae from fish with total length: A. 50 mm; B. 109 mm; C. 157 $\mathrm{mm}$. The medial face is shown in A1; $\mathrm{B} 1$; $\mathrm{C} 1$; the lateral face in A2; B2; C2; and the ventral profile in A3; B3; C3 (Illustration: Michelle Konig; Photos: Cesar Santificetur). 
A1

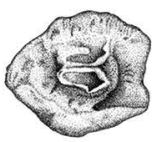

A2

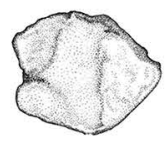

A3
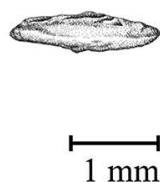

B1

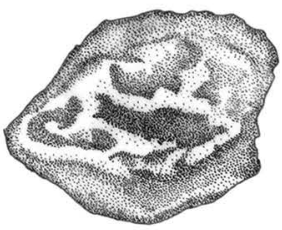

B2

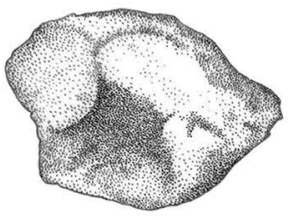

B3
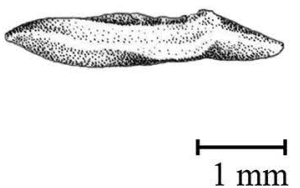

C1

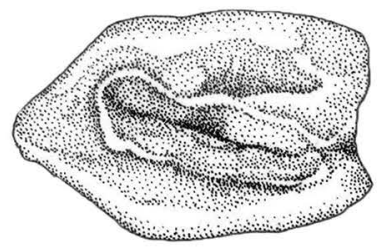

C2

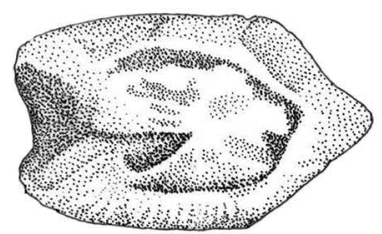

C3

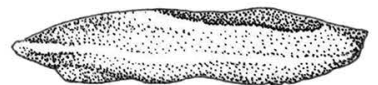

$\stackrel{\longmapsto}{1 \mathrm{~mm}}$
A1

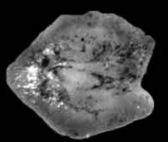

A2

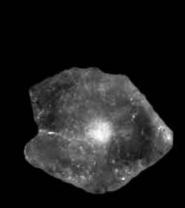

A3
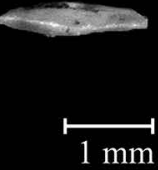

B1

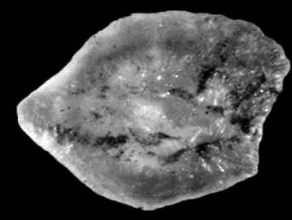

B2

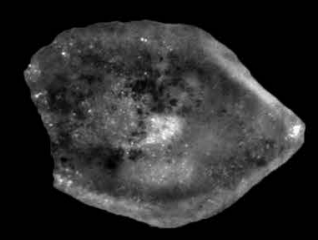

B3

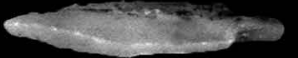

$1 \mathrm{~mm}$
C1

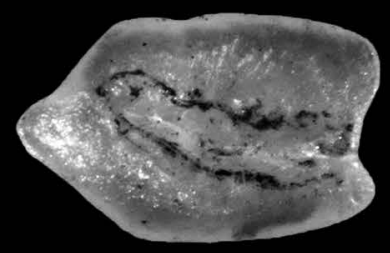

C2

C3
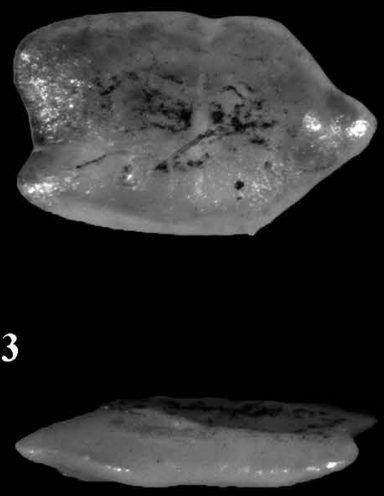

Plate 10. Illustrations (above) and photographs (below) of Etropus crossotus right sagittae from fish with total length: A. $50 \mathrm{~mm}$; B. $109 \mathrm{~mm}$; C. $157 \mathrm{~mm}$. The medial face is shown in A1; 1 1; C1; the lateral face in A2; 32 ; 2 ; and the ventral profile in A3; B3; C3 (Illustration: Michelle Konig; Photos: Cesar Santificetur). 
A1

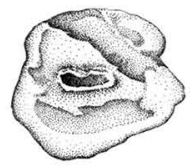

A2

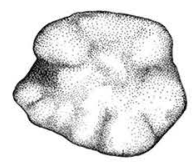

A3

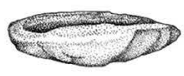

$\longmapsto 1 \mathrm{~mm}$
B1

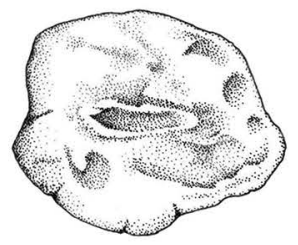

B2

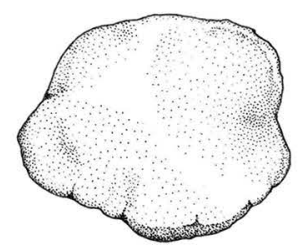

B3

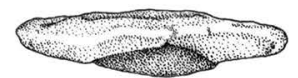

1
C1

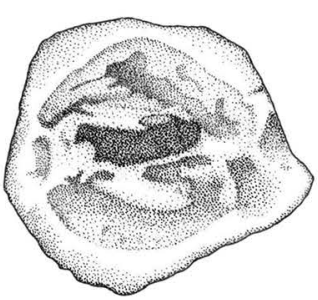

C2

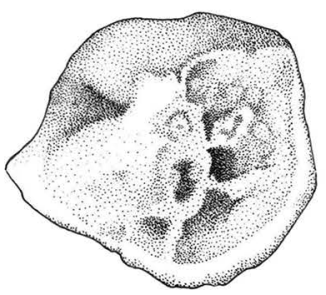

C3

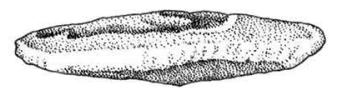

$\longmapsto 1 \mathrm{~mm}$

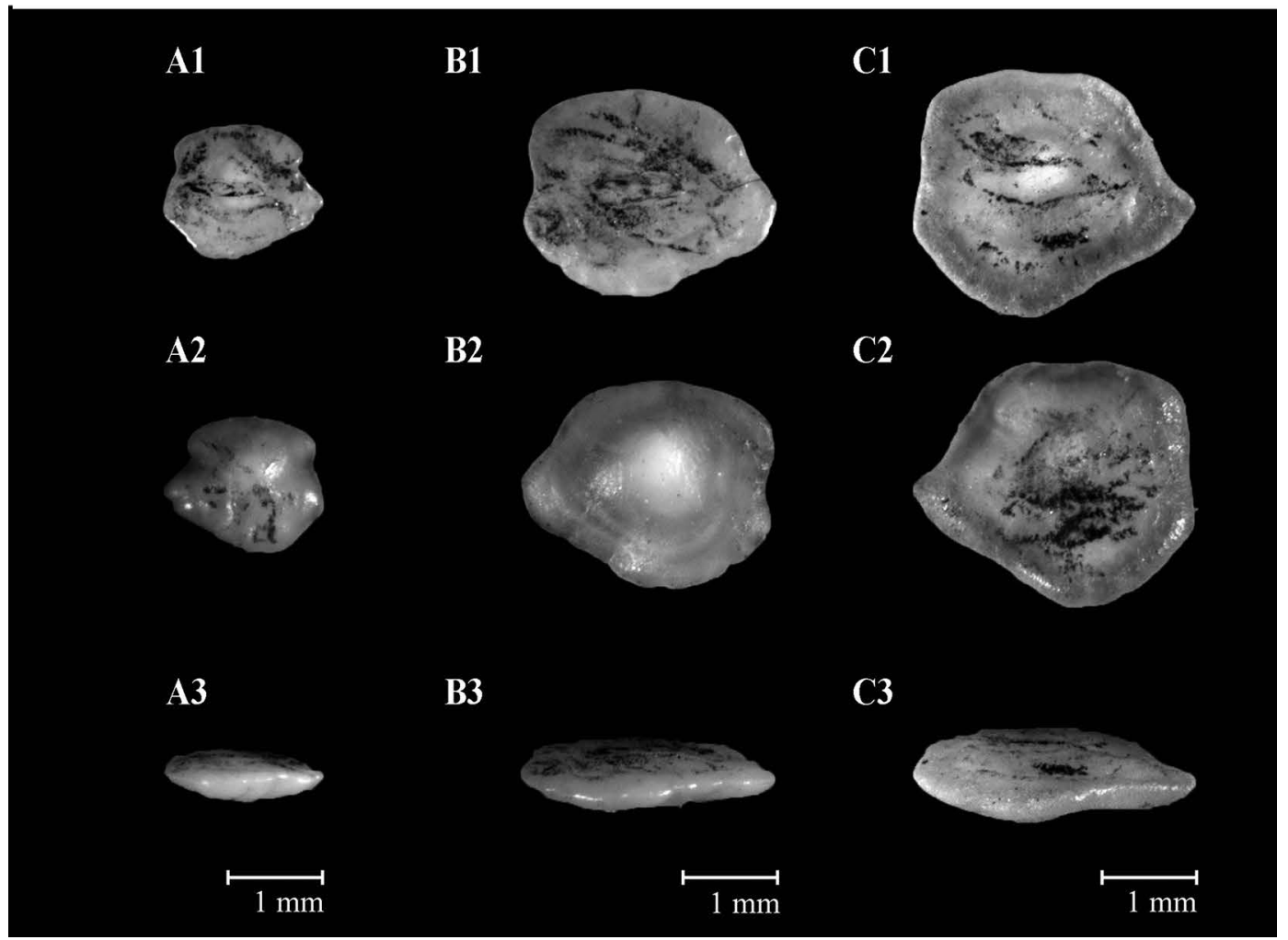

Plate 11. Illustrations (above) and photographs (below) of Etropus longimanus left sagittae from fish with total length: A. $53 \mathrm{~mm}$; B. $98 \mathrm{~mm}$; C. $145 \mathrm{~mm}$. The medial face is shown in $\mathrm{A} 1 ; \mathrm{B} 1 ; \mathrm{C} 1$; the lateral face in $\mathrm{A} 2 ; \mathrm{B} 2 ; \mathrm{C} 2$; and the ventral profile in A3; B3; C3 (Illustration: Michelle Konig; Photos: Cesar Santificetur). 
A1

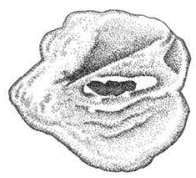

A2

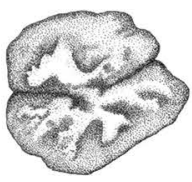

A3

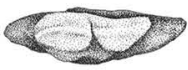

B1

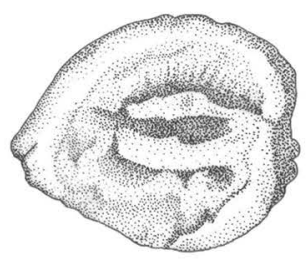

B2

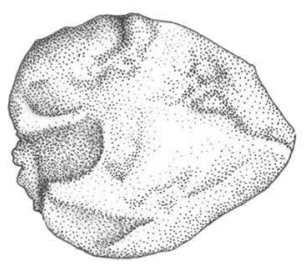

B3
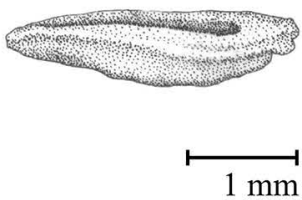

C1

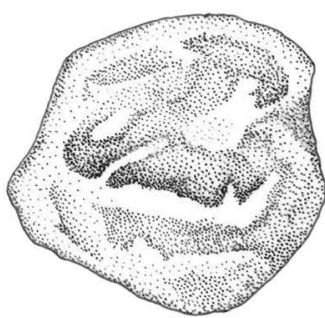

C2

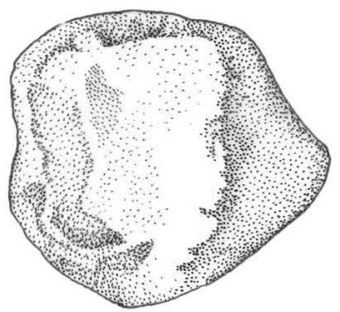

C3

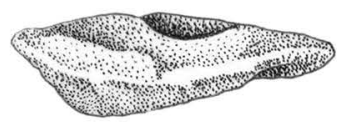

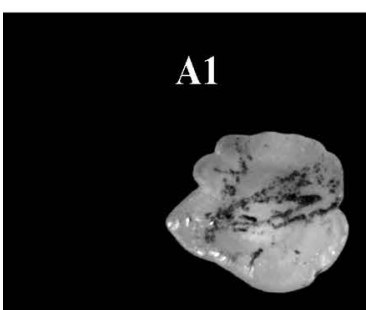

A2

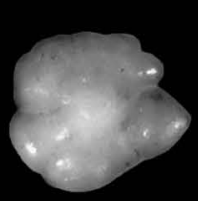

A3

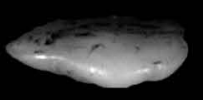

$1 \mathrm{~mm}$
B1

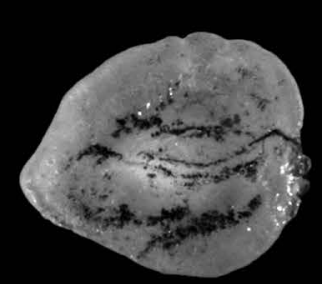

B2

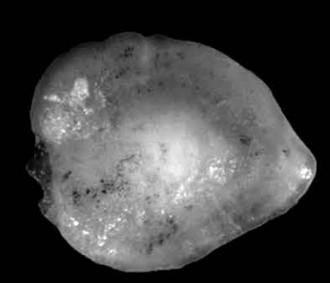

B3
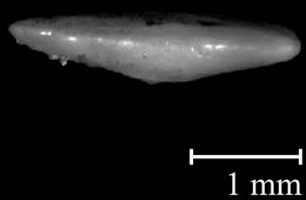

C1

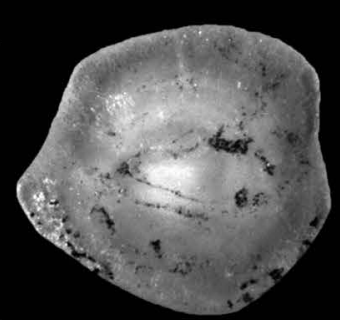

C2

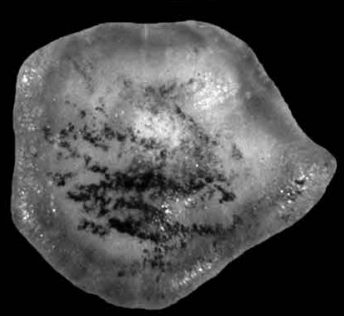

C3

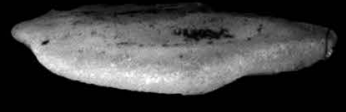

$1 \mathrm{~mm}$

Plate 12. Illustrations (above) and photographs (below) of Etropus longimanus right sagittae from fish with total length: A. $53 \mathrm{~mm}$; B. $98 \mathrm{~mm}$; C. $145 \mathrm{~mm}$. The medial face is shown in $\mathrm{A} 1 ; \mathrm{B} 1 ; \mathrm{C} 1$; the lateral face in $\mathrm{A} 2 ; \mathrm{B} 2 ; \mathrm{C} 2$; and the ventral profile in A3; B3; C3 (Illustration: Michelle Konig; Photos: Cesar Santificetur). 
A1

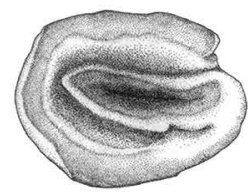

A2

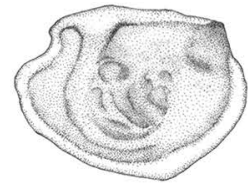

A3

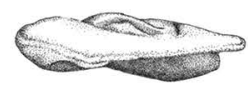

$1 \stackrel{1}{\mathrm{~mm}}$
B1

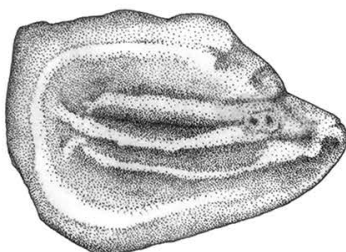

B2

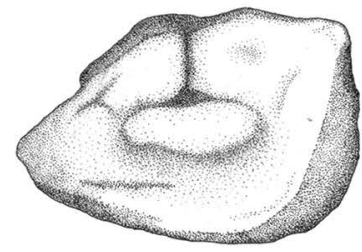

B3

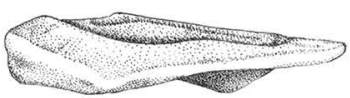

$\stackrel{\longmapsto}{\longmapsto}$

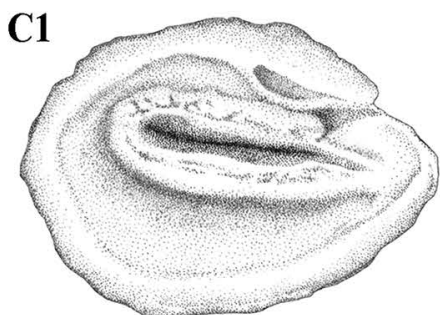

C2

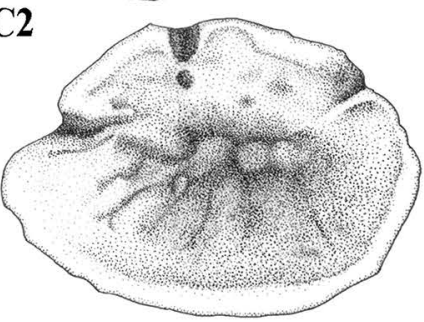

C3

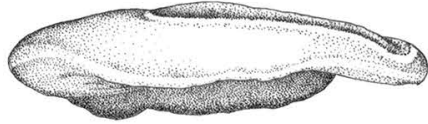

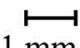
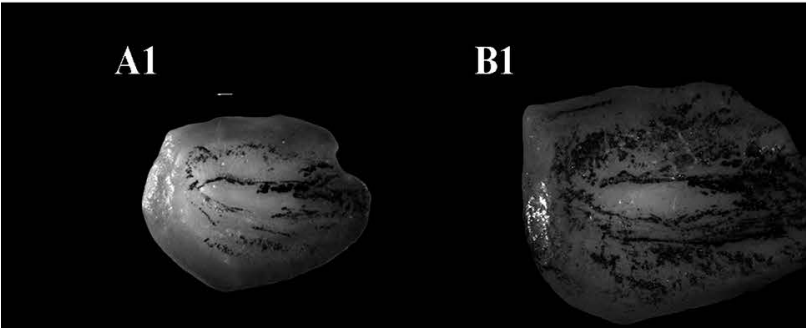

\section{C1}

A2

B2

\section{C2}
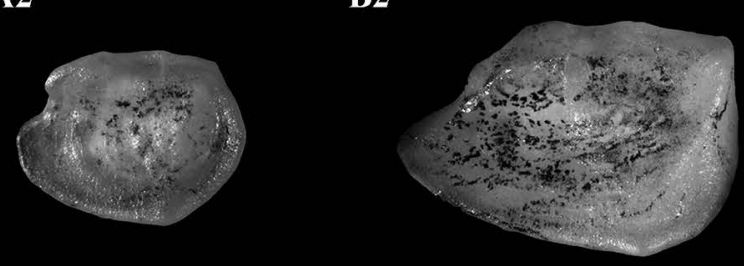

A3

B3

C3
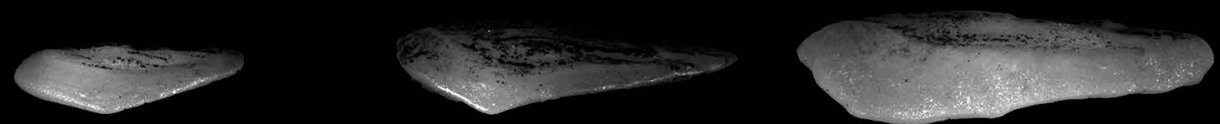

$1 \stackrel{\longmapsto m}{\mathrm{~mm}}$
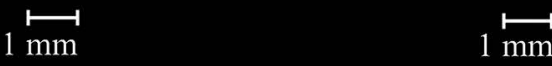

Plate 13. Illustrations (above) and photographs (below) of Paralichthys isosceles left sagittae from fish with total length: A. $197 \mathrm{~mm}$; B. $287 \mathrm{~mm}$; C. $377 \mathrm{~mm}$. The medial face is shown in $\mathrm{A} 1 ; \mathrm{B} 1 ; \mathrm{C} 1$; the lateral face in $\mathrm{A} 2 ; \mathrm{B} 2 ; \mathrm{C} 2$; and the ventral profile in $\mathrm{A} 3$; $\mathrm{B} 3$; $\mathrm{C} 3$ (Illustration: Michelle Konig; Photos: Cesar Santificetur). 
A1

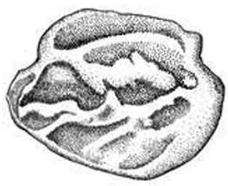

A2

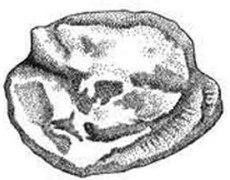

A3

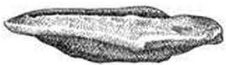

$2 \mathrm{~mm}$
B1

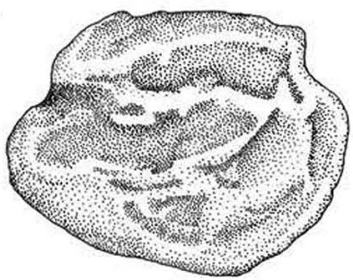

B2

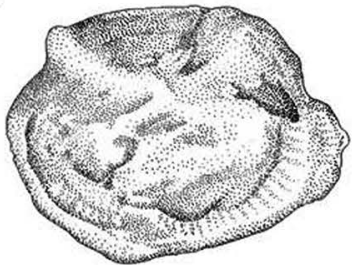

B3
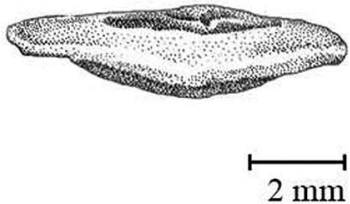

C1

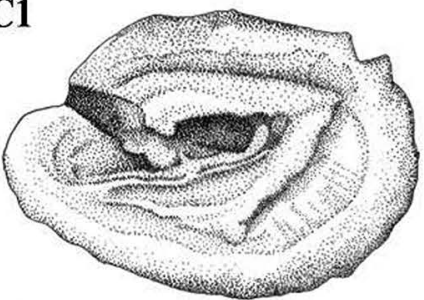

C2

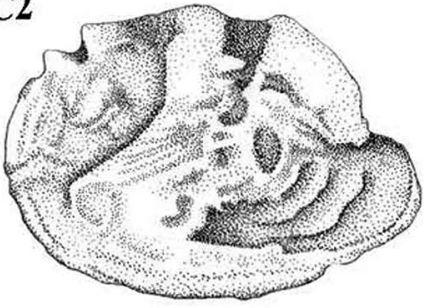

C3

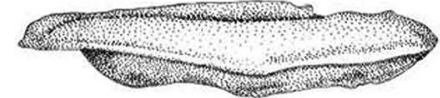

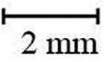

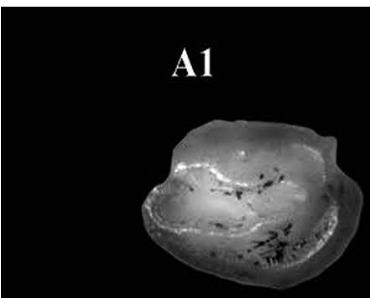

B1

A2

B2

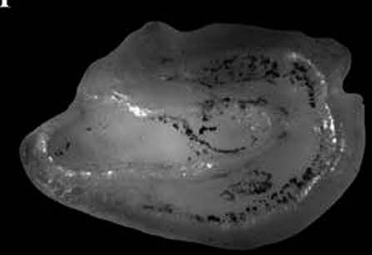

C1
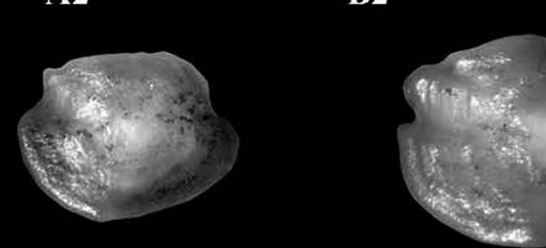

C2

A3

B3

C3
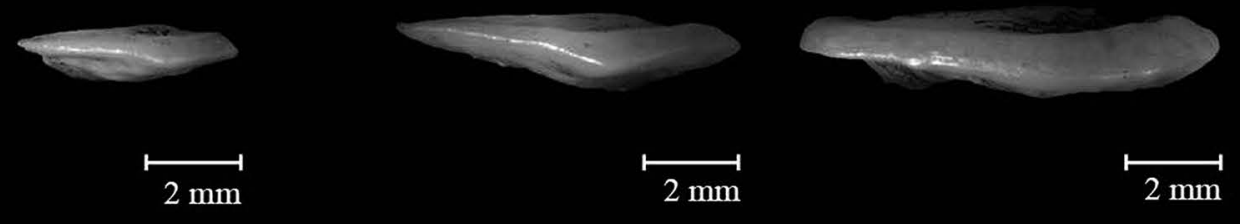

Plate 14. Illustrations (above) and photographs (below) of Paralichthys isosceles right sagittae from fish with total length: A. $197 \mathrm{~mm}$; B. $287 \mathrm{~mm}$; C. $377 \mathrm{~mm}$. The medial face is shown in A1; B1; C1; the lateral face in A2; B2; C2; and the ventral profile in A3; B3; C3 (Illustration: Michelle Konig; Photos: Cesar Santificetur). 
A1

ब्as?

A2

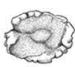

A3

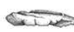

$\stackrel{\longmapsto}{\mathrm{mm}}$
B1

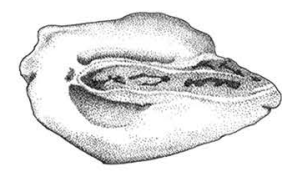

B2

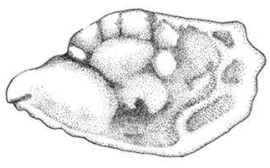

B3

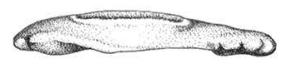

$\stackrel{\longmapsto}{2 \mathrm{~mm}}$

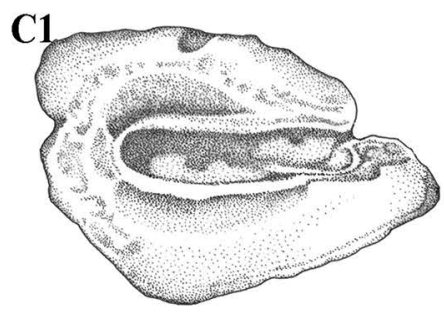

$\mathrm{C} 2$

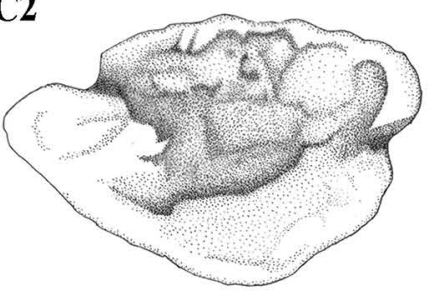

C3

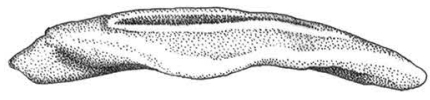

$\stackrel{\longmapsto}{2 \mathrm{~mm}}$

A1

B1

C1

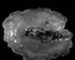

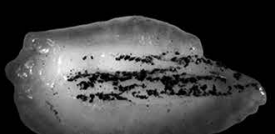

$\mathbf{A 2}$

B2

C2
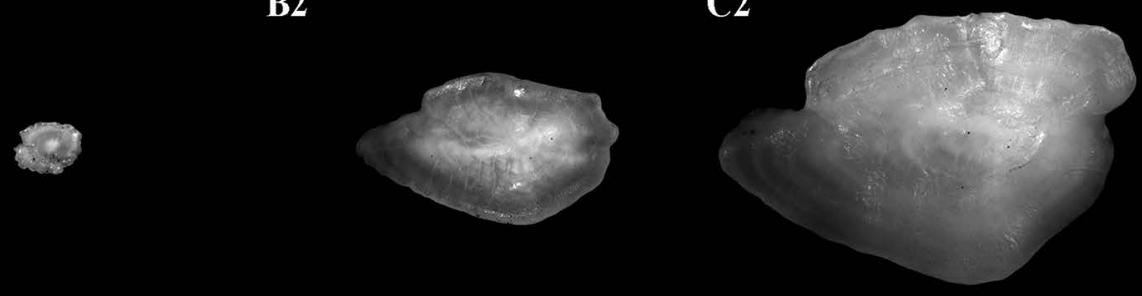

A3

B3

C3

wasass
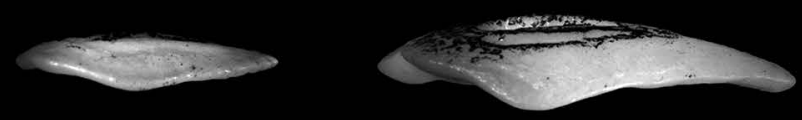

1 mm
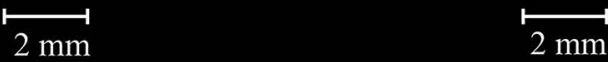

Plate 15. Illustrations (above) and photographs (below) of Paralichthys patagonicus left sagittae from fish with total length: A. $125 \mathrm{~mm}$; B. 323 $\mathrm{mm}$; C. $555 \mathrm{~mm}$. The medial face is shown in A1; B1; C1; the lateral face in A2; B2; C2; and the ventral profile in A3; B3; C3 (Illustration: Michelle Konig; Photos: Cesar Santificetur). 
A1

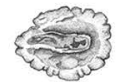

A2

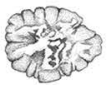

A3

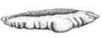

$\stackrel{\mathrm{mm}}{\longrightarrow}$
B1

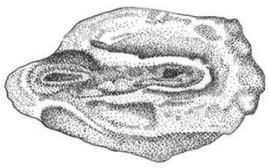

B2

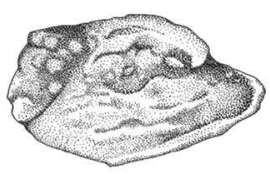

B3
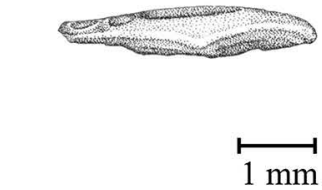

C1

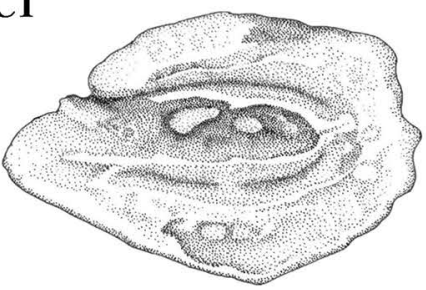

C2

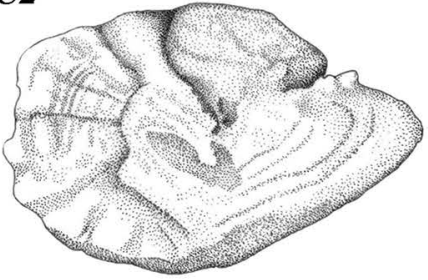

C3

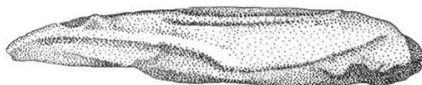

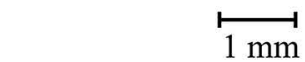
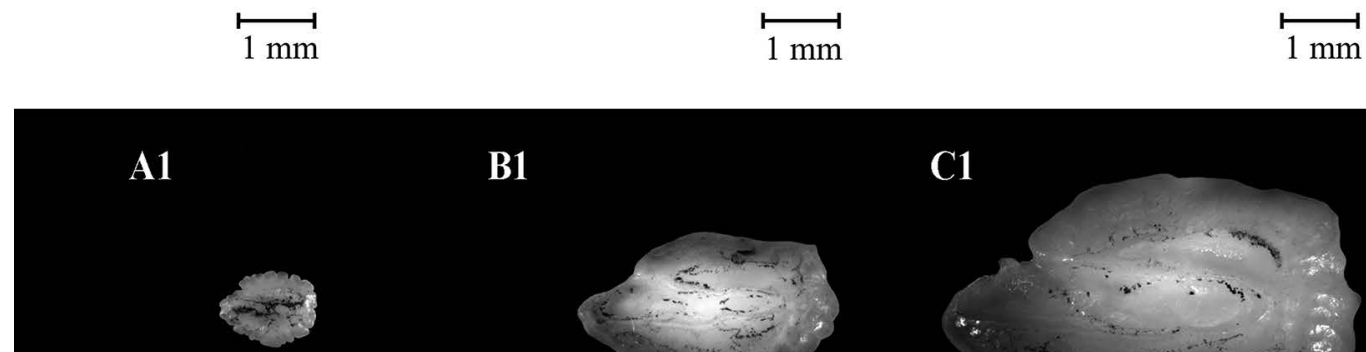

B1

C1
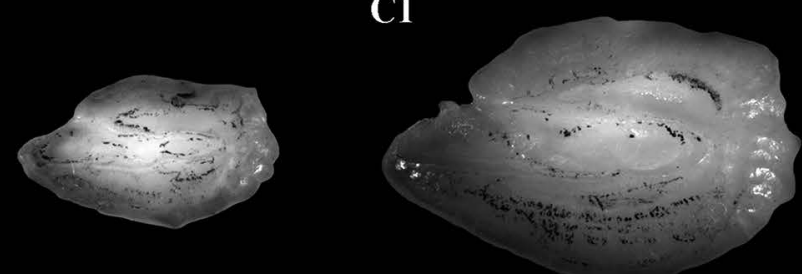

A2

B2

C2
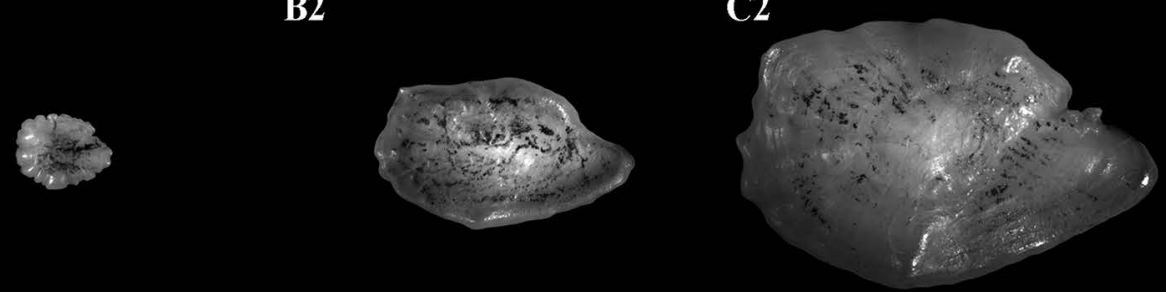

A3

B3

C3
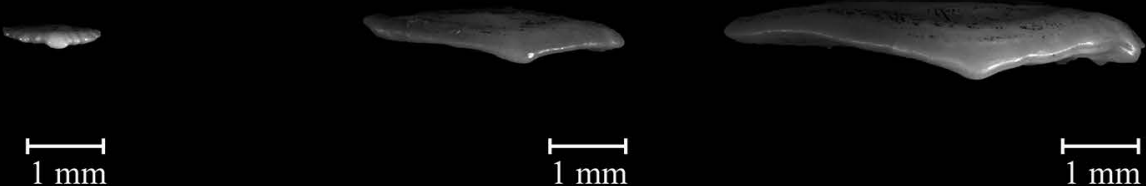

$1 \mathrm{~mm}$

$\stackrel{\mathrm{mm}}{\longmapsto}$

Plate 16. Illustrations (above) and photographs (below) of Paralichthys patagonicus right sagittae from fish with total length: A. $125 \mathrm{~mm}$; B. 323 $\mathrm{mm}$; C. $555 \mathrm{~mm}$. The medial face is shown in A1; 1 1; 1 ; the lateral face in A2; B2; C2; and the ventral profile in A3; B3; C3 (Illustration: Michelle Konig; Photos: Cesar Santificetur). 
A1

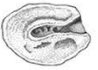

A2

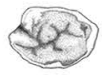

A3

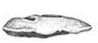

$\stackrel{\longmapsto}{\mathrm{mm}}$
B1

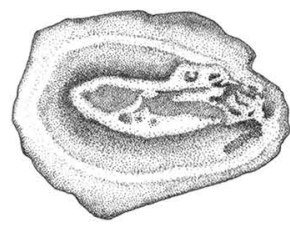

B2

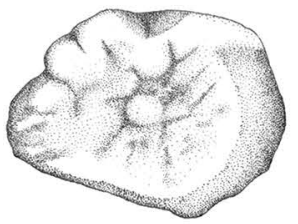

B3

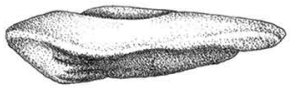

$1 \stackrel{\mathrm{mm}}{\longmapsto}$

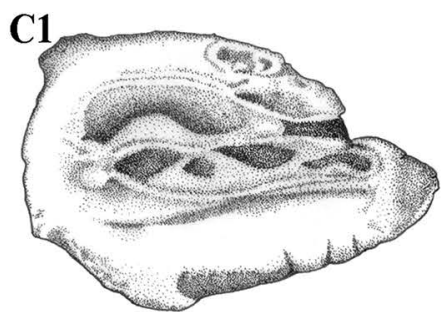

C2

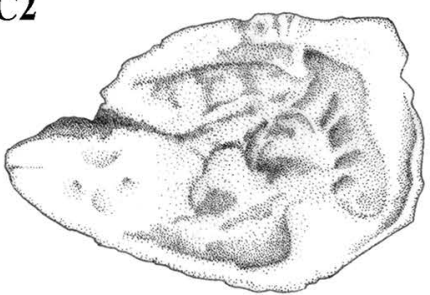

C3

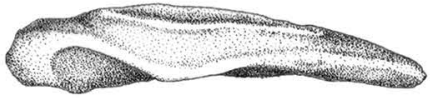

$\longmapsto$

\section{A1}

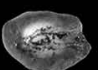

A2

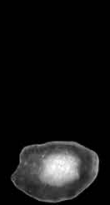

A3

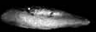

cyeres

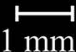

B1

B2
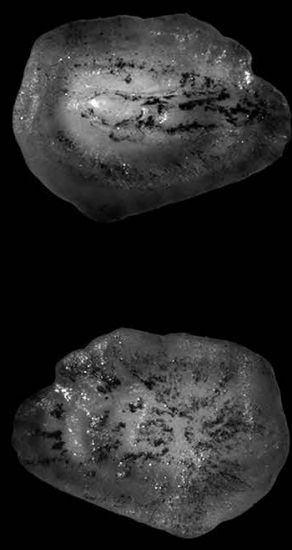

B3

C2

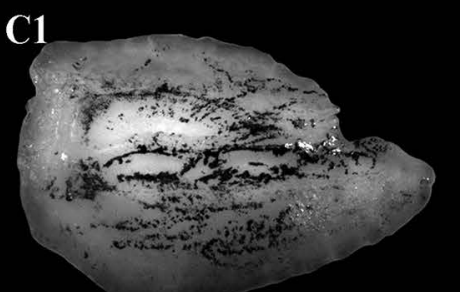

C3
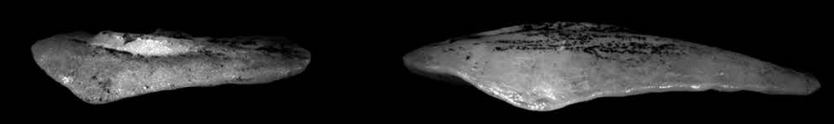
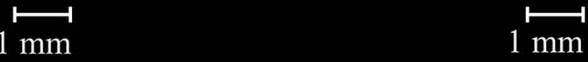

Plate 17. Illustrations (above) and photographs (below) of Paralichthys triocellatus left sagittae from fish with total length: A. 70 mm; B. 205 mm; C. $338 \mathrm{~mm}$. The medial face is shown in A1; B1; C1; the lateral face in A2; B2; C2; and the ventral profile in A3; B3; C3 (Illustration: Michelle Konig; Photos: Cesar Santificetur). 
A1

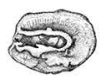

A2

A3

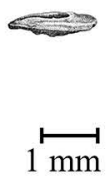

B1

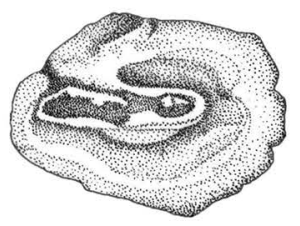

B2

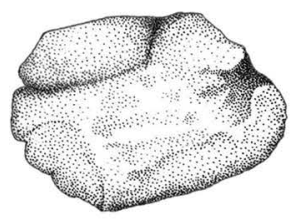

B3

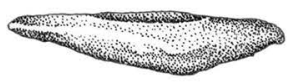

$\stackrel{\longmapsto}{1 \mathrm{~mm}}$
C1

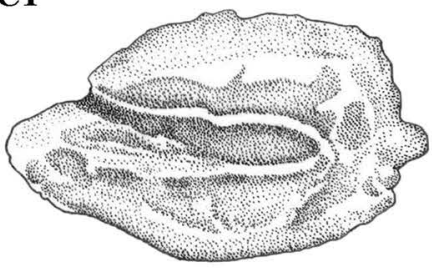

C2

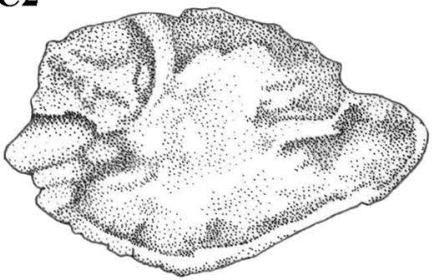

C3

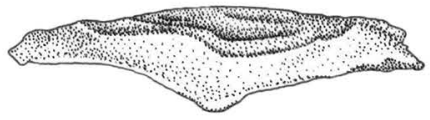

A1

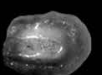

A2

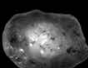

A3

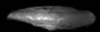

$1 \mathrm{~mm}$
B1

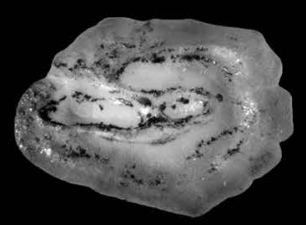

B2

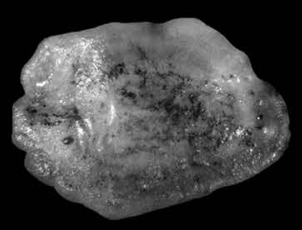

B3

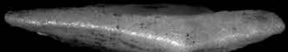

C1

C2

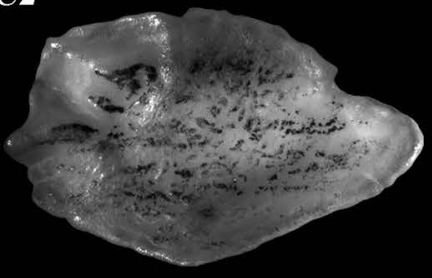

C3

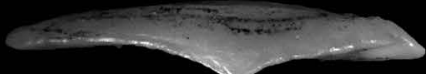

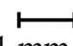


A1

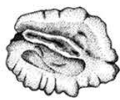

A2

A3

a)

$1 \stackrel{\mathrm{mm}}{\longmapsto}$
B1

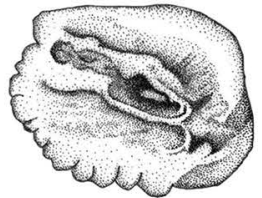

B2

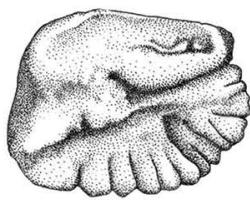

B3

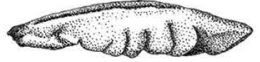

$1 \stackrel{\mathrm{mm}}{ }$

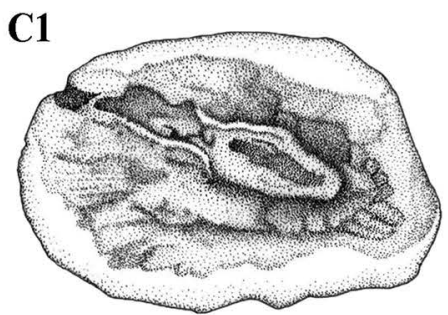

C2

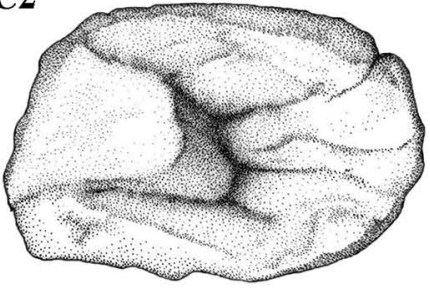

C3

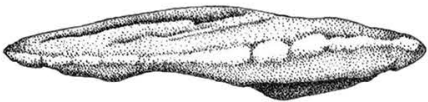

$1 \stackrel{\mathrm{mm}}{ }$
A1

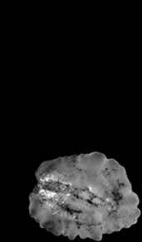

A2

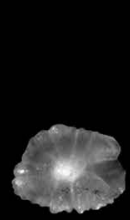

A3

- 6979
B1

B2

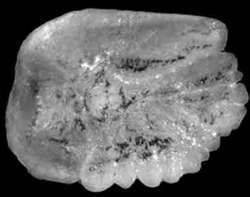

B3

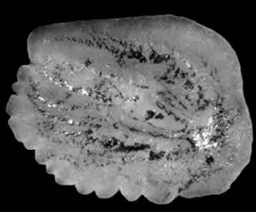

C1

C2

C3
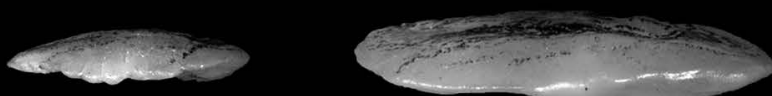

$1 \mathrm{~mm}$

Plate 19. Illustrations (above) and photographs (below) of Syacium papillosum left sagittae from fish with total length: A. $60 \mathrm{~mm}$; B. $162 \mathrm{~mm}$; C. $275 \mathrm{~mm}$. The medial face is shown in $\mathrm{A} 1 ; \mathrm{B} 1 ; \mathrm{C} 1$; the lateral face in $\mathrm{A} 2 ; \mathrm{B} 2 ; \mathrm{C} 2$; and the ventral profile in A3; B3; C3 (Illustration: Michelle Konig; Photos: Cesar Santificetur). 
A1

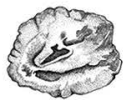

A2

A3

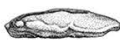

$1 \mathrm{~mm}$
B1

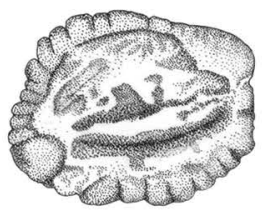

B2

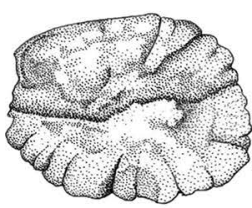

B3

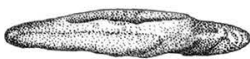

$1 \mathrm{~mm}$

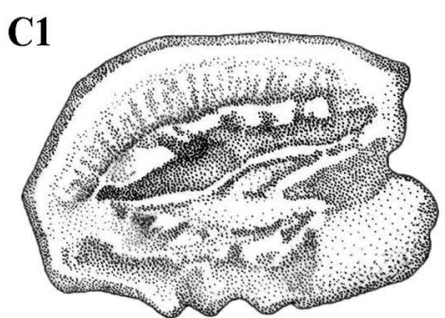

C2

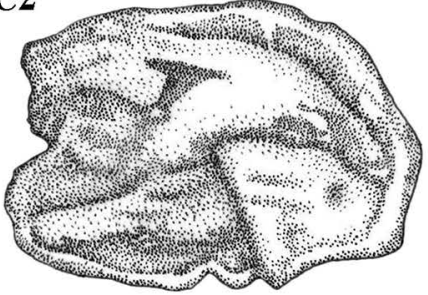

C3

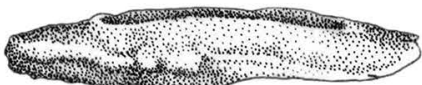

$1 \stackrel{\mathrm{mm}}{\longmapsto}$

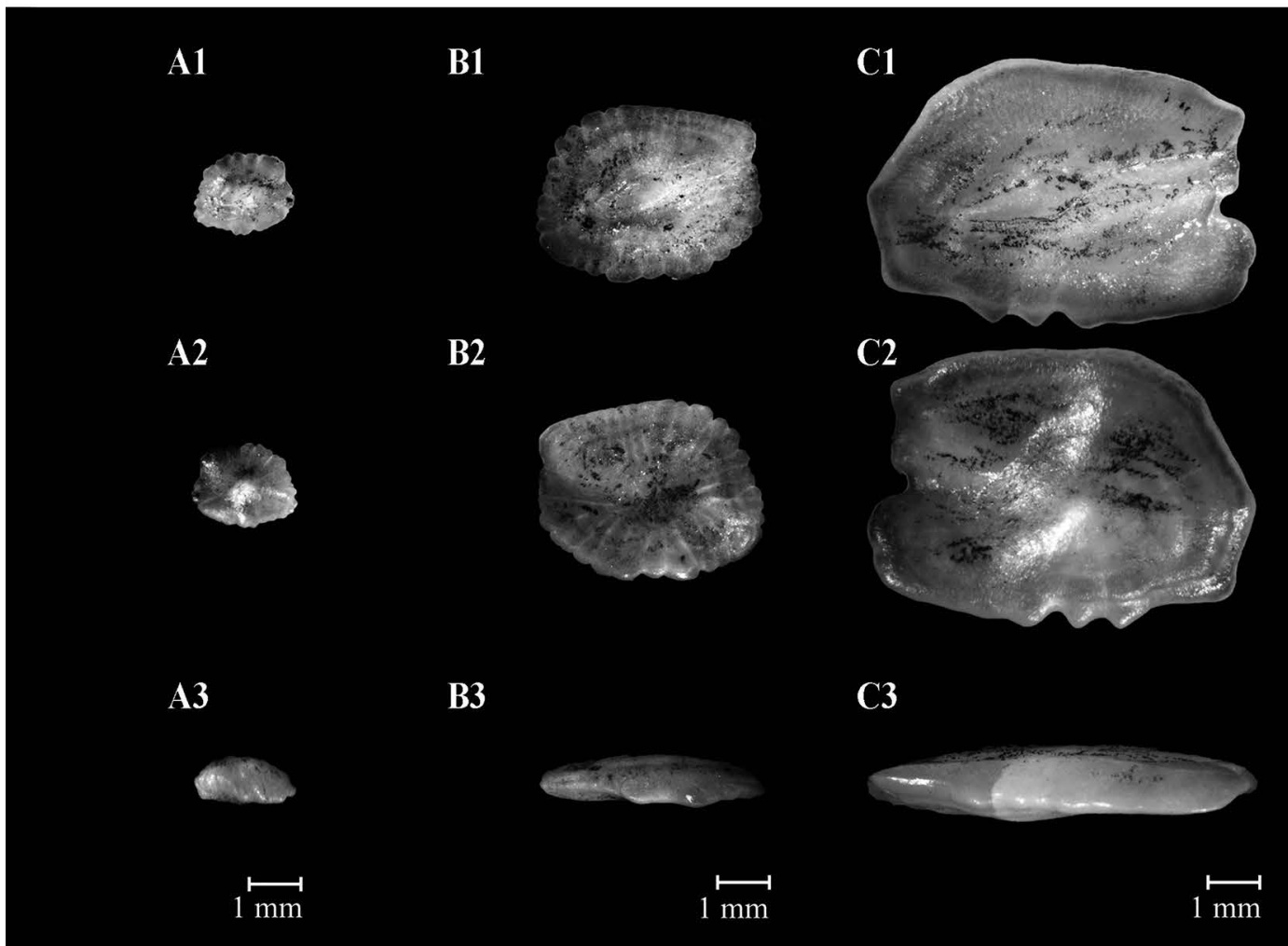

Plate 20. Illustrations (above) and photographs (below) of Syacium papillosum right sagittae from fish with total length: A. $60 \mathrm{~mm}$; B. $162 \mathrm{~mm}$; C. $275 \mathrm{~mm}$. The medial face is shown in A1; B1; C1; the lateral face in A2; B2; C2; and the ventral profile in A3; B3; C3 (Illustration: Michelle Konig; Photos: Cesar Santificetur). 
A1

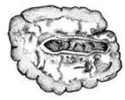

A2

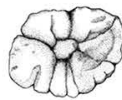

A3

(1) 225

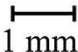

B1

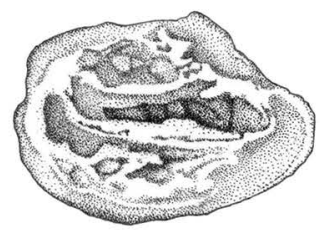

B2

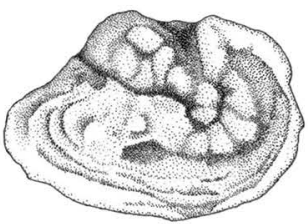

B3

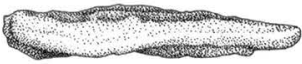

$\stackrel{\longmapsto}{\mathrm{mm}}$
C1

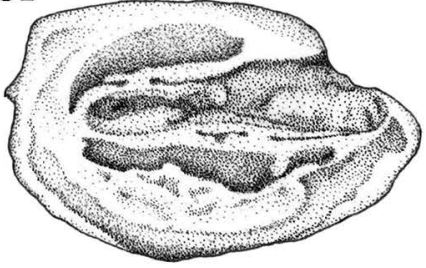

C2

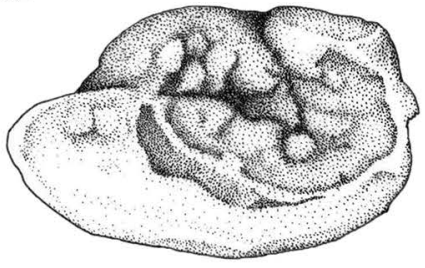

C3

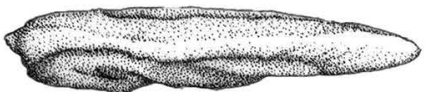

$1 \mathrm{~mm}$
A1

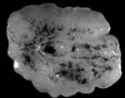

A2

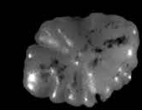

A3

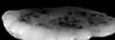

$\stackrel{\longmapsto}{\mathrm{mm}}$
B1

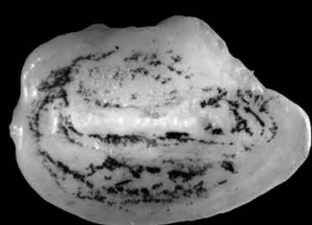

B2

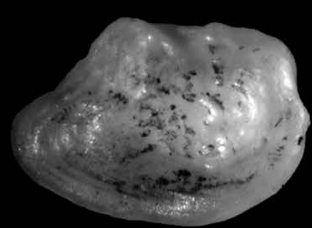

B3

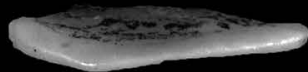

$1 \mathrm{~mm}$
C1

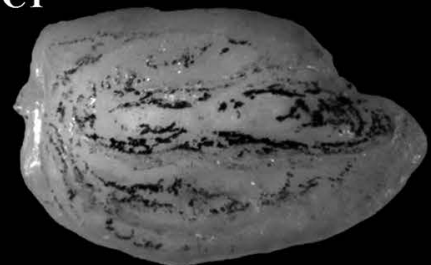

C2

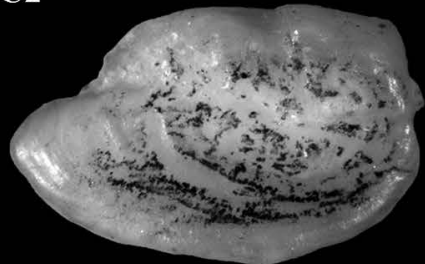

C3

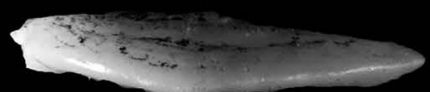

Plate 21. Illustrations (above) and photographs (below) of Xystreurys rasile left sagittae from fish with total length: A. 76 mm; B. 248 mm; C. 392 $\mathrm{mm}$. The medial face is shown in A1; $\mathrm{B} 1 ; \mathrm{C} 1$; the lateral face in A2; $\mathrm{B} 2$; $\mathrm{C} 2$; and the ventral profile in A3; B3; C3 (Illustration: Michelle Konig; Photos: Cesar Santificetur). 
A1

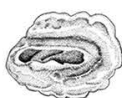

A2

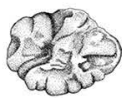

A3

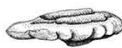

$\stackrel{\longmapsto}{\mathrm{mm}}$
B1

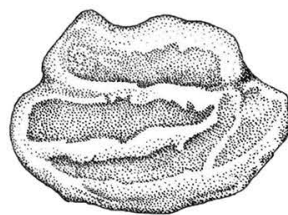

B2

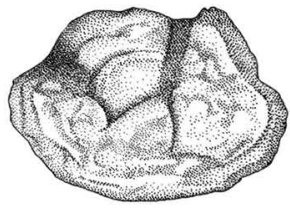

B3

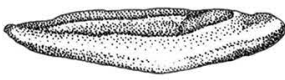

$\longmapsto$
C1

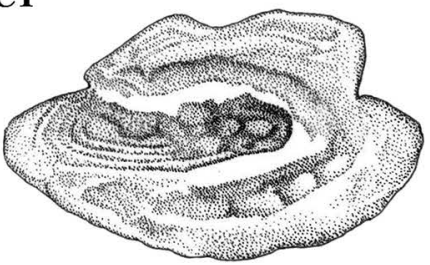

C2

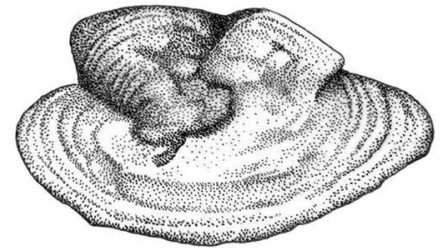

C3

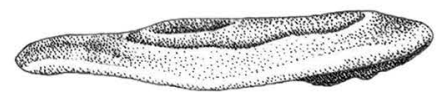

$1 \longmapsto$

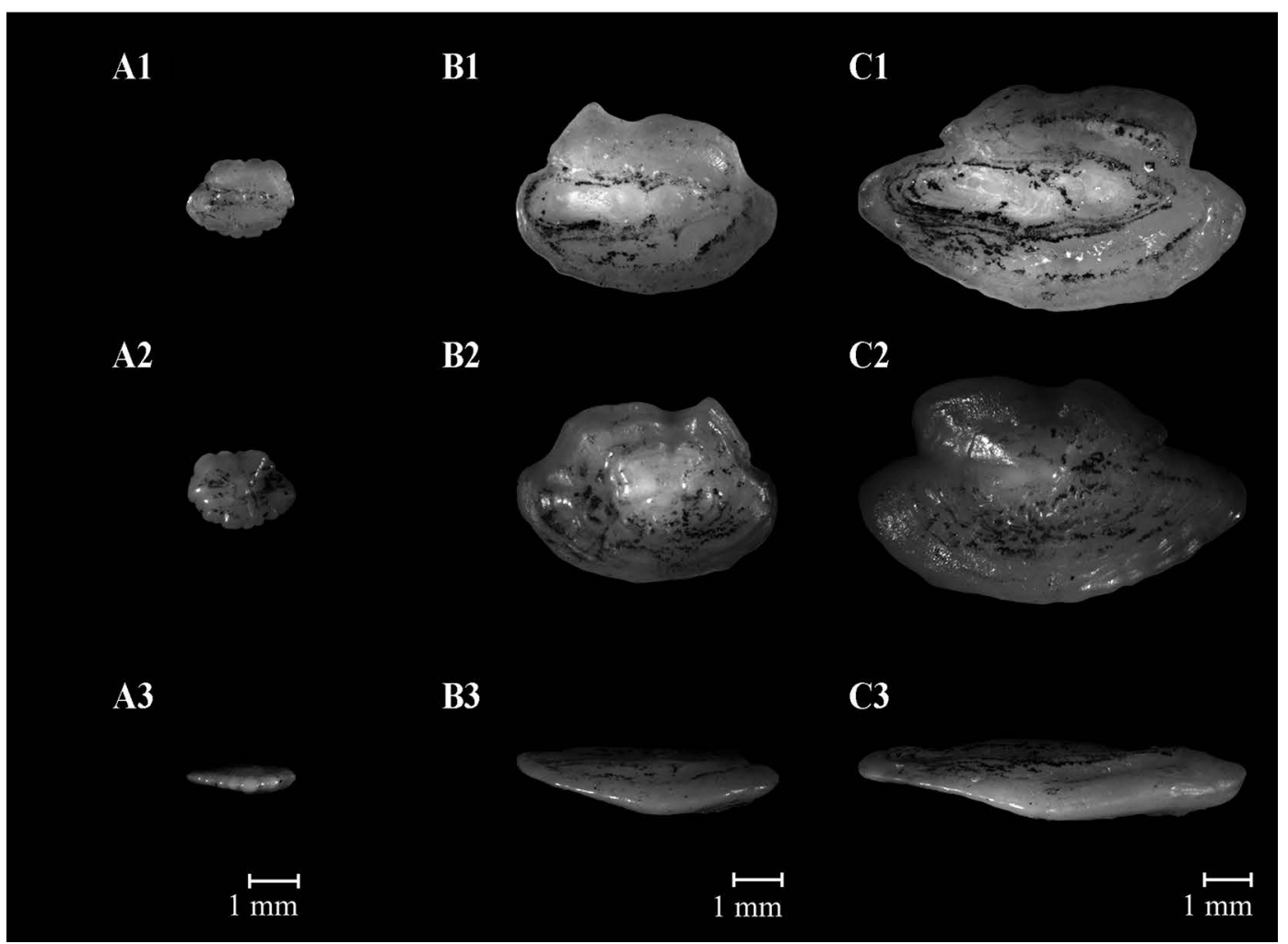

Plate 22. Illustrations (above) and photographs (below) of Xystreurys rasile right sagittae from fish with total length: A. 76 mm; B. 248 mm; C. 392 $\mathrm{mm}$. The medial face is shown in $\mathrm{A} 1$; $\mathrm{B} 1$; $\mathrm{C} 1$; the lateral face in $\mathrm{A} 2$; $\mathrm{B} 2$; $\mathrm{C} 2$; and the ventral profile in A3; 33 ; $\mathrm{C} 3$ (Illustration: Michelle Konig; Photos: Cesar Santificetur). 
A1

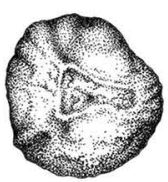

A2

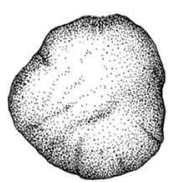

A3

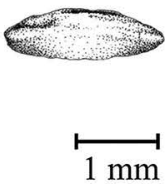

B1

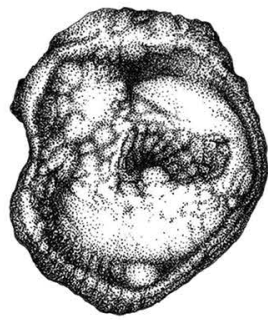

B2

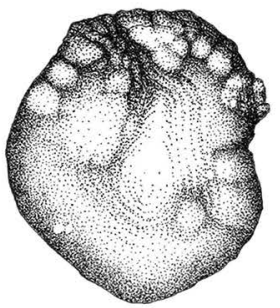

B3

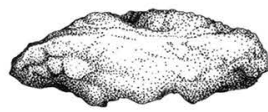

$\longmapsto 1 \mathrm{~mm}$
A1

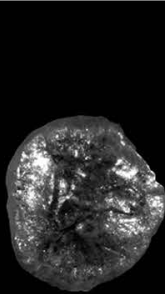

A2

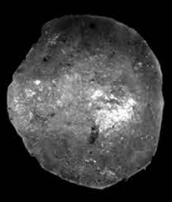

A3
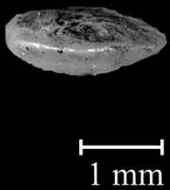

B1

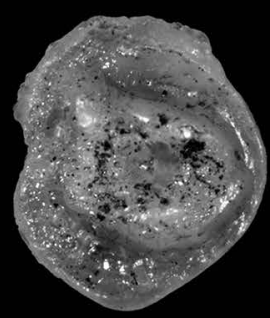

B2

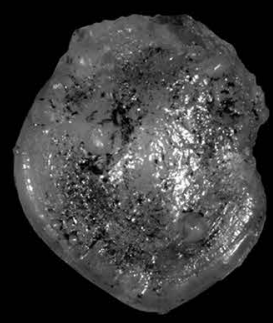

B3

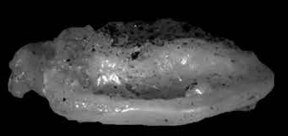

Plate 23. Illustrations (above) and photographs (below) of Symphurus tessellatus left sagittae from fish with total length: A. 137 mm; B. 218 mm. The medial face is shown in A1; B1; the lateral face in A2; B2; and the ventral profile in A3; B3 (Illustration: Alexandre Arackawa; Photos: Cesar Santificetur). 
A1

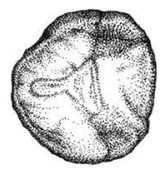

A2

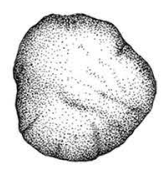

A3

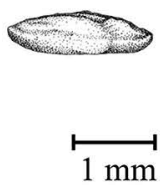

A1

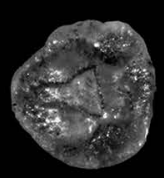

A2

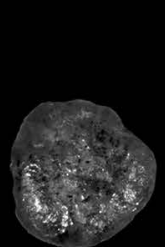

A3
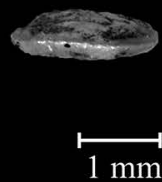

B1

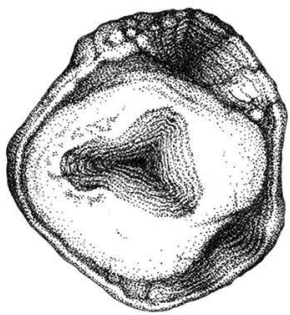

B2

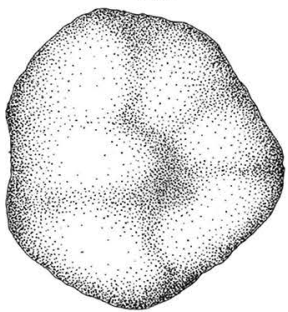

B3

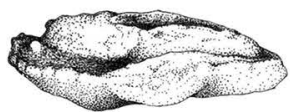

$1 \mathrm{~mm}$

B1

B2
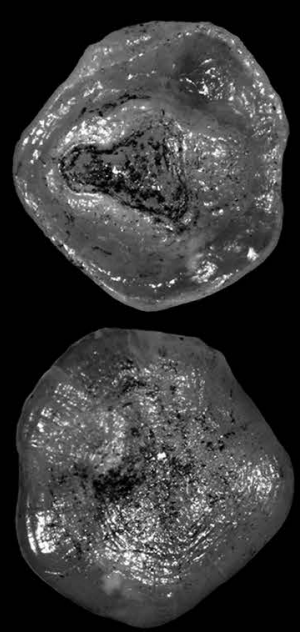

B3

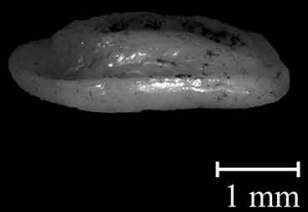

Plate 24. Illustrations (above) and photographs (below) of Symphurus tessellatus right sagittae from fish with total length: A. $137 \mathrm{~mm}$; B. $218 \mathrm{~mm}$. The medial face is shown in A1; B1; the lateral face in A2; B2; and the ventral profile in A3; B3 (Illustration: Alexandre Arackawa; Photos: Cesar Santificetur). 
A1

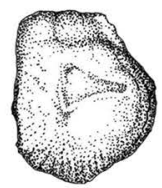

A2

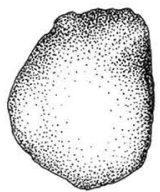

A3
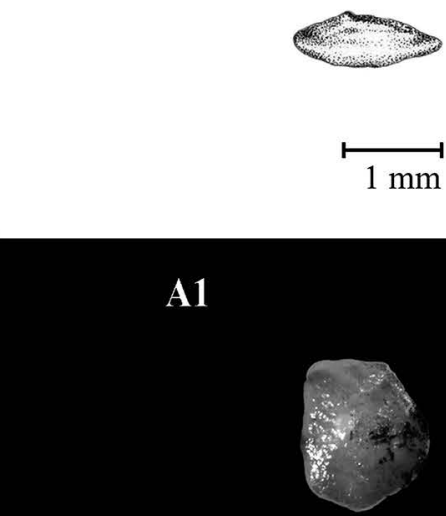

A2

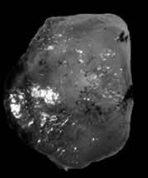

B2

B1
B3
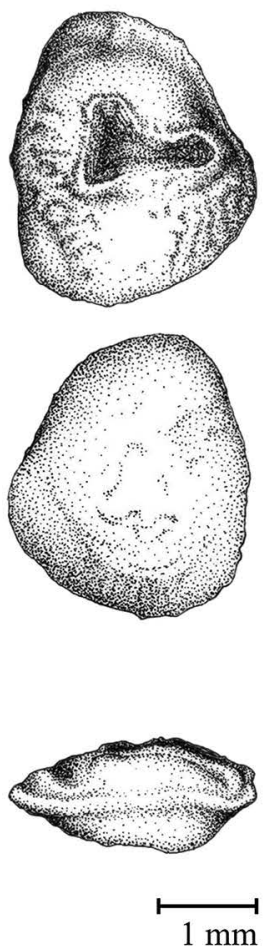

B2

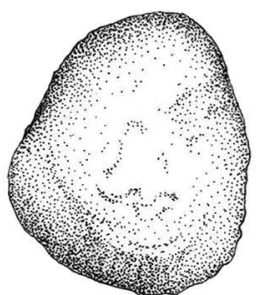

$1 \mathrm{~mm}$ 
A1

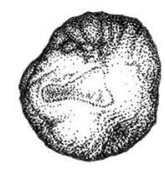

A2

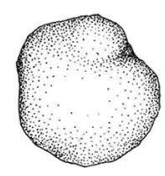

A3

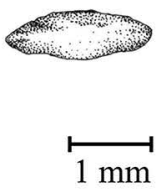

B1

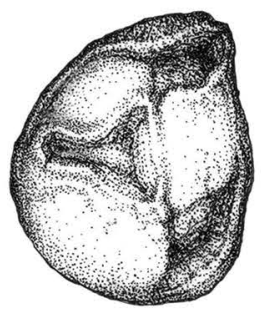

B2

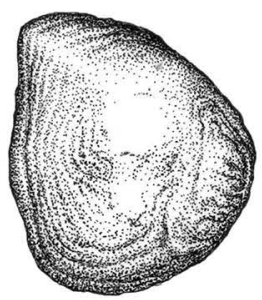

B3

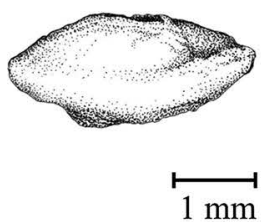

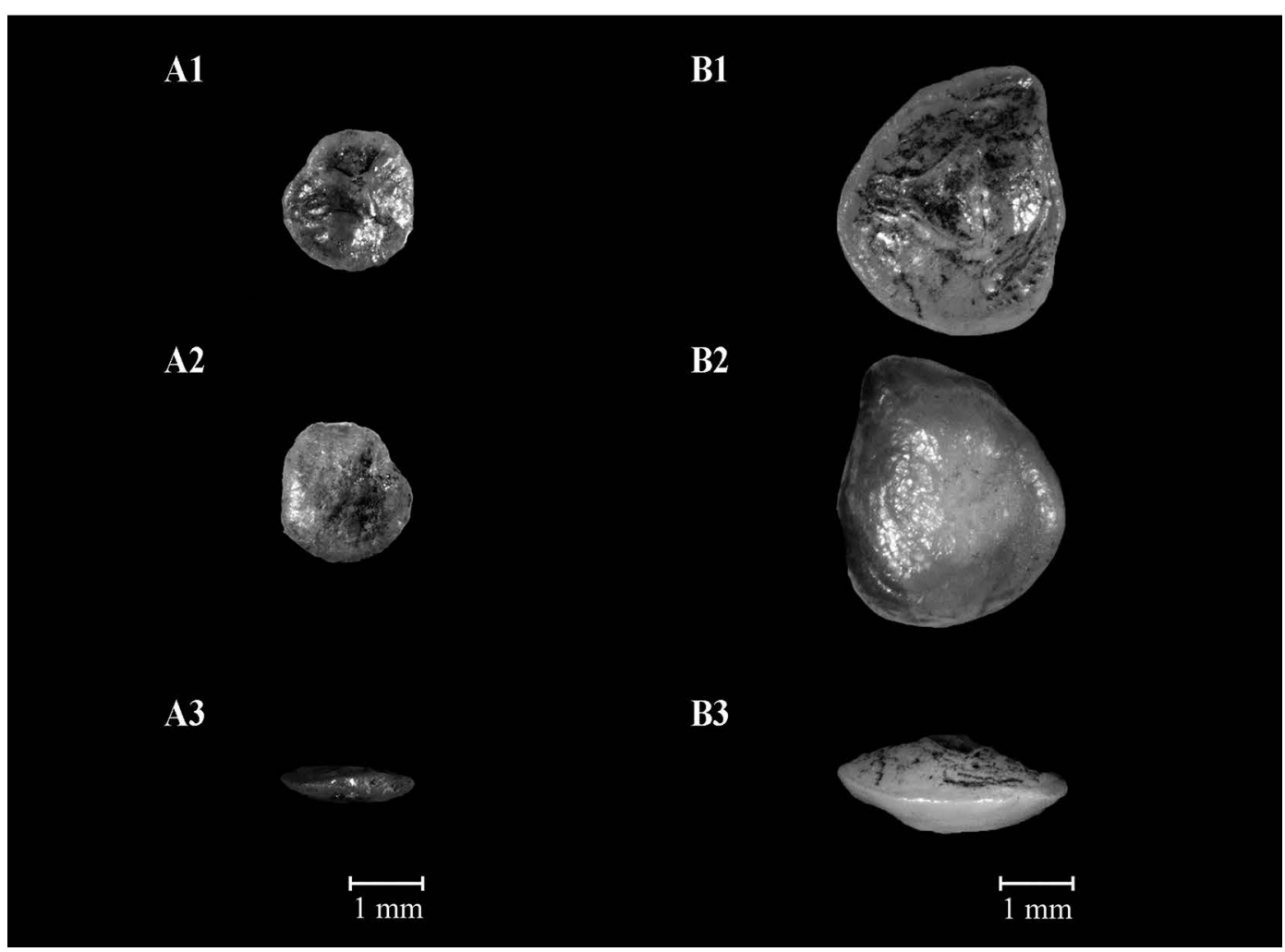

Plate 26. Illustrations (above) and photographs (below) of Symphurus jenynsi right sagittae from fish with total length: A. $128 \mathrm{~mm}$; B. $231 \mathrm{~mm}$. The medial face is shown in A1; B1; the lateral face in A2; B2; and the ventral profile in A3; B3 (Illustration: Alexandre Arackawa; Photos: Cesar Santificetur). 
A1

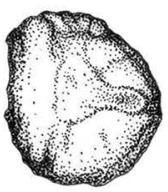

A2

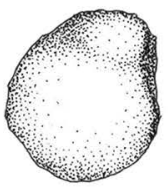

A3
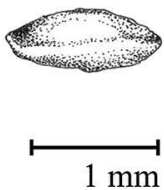

B1

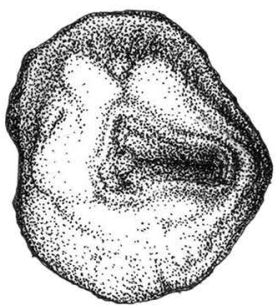

B2

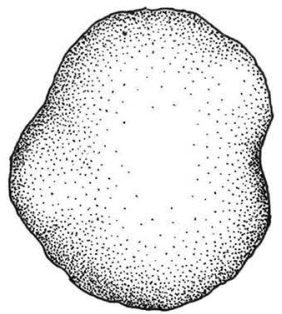

B3

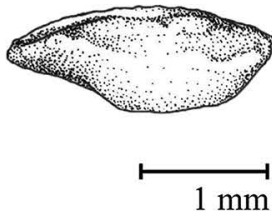

\section{B1}

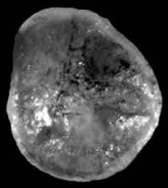

A2

B2
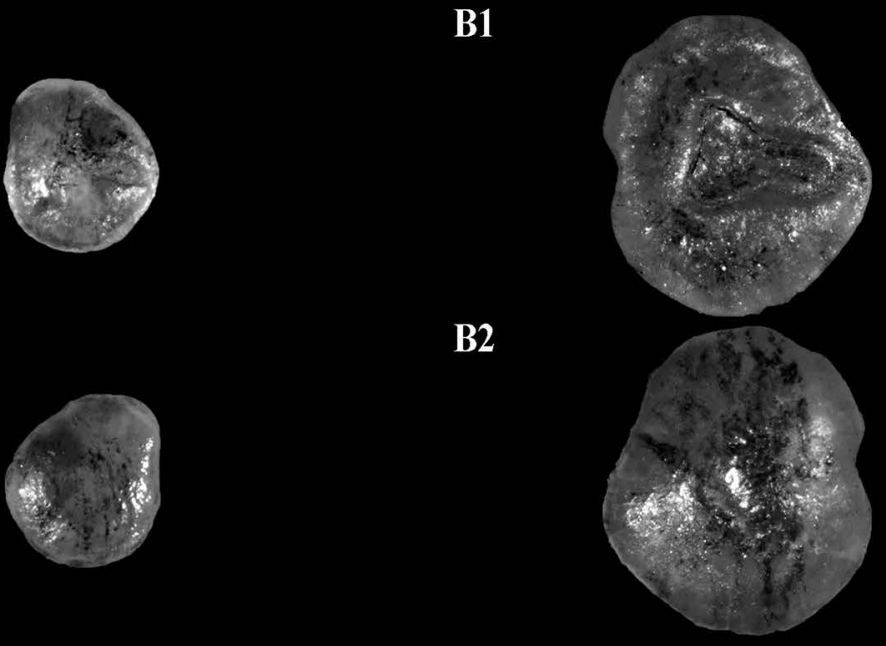

A3

B3

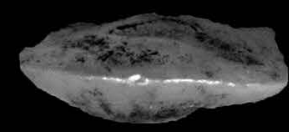

$1 \mathrm{~mm}$

Plate 27. Illustrations (above) and photographs (below) of Symphurus plagusia left sagittae from fish with total length: A. 113 mm; B. $204 \mathrm{~mm}$. The medial face is shown in A1; B1; the lateral face in A2; B2; and the ventral profile in A3; B3 (Illustration: Alexandre Arackawa; Photos: Cesar Santificetur). 
A1

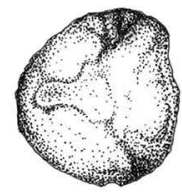

A2

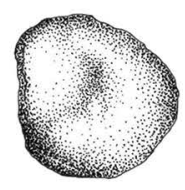

A3
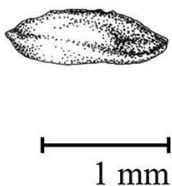

B1

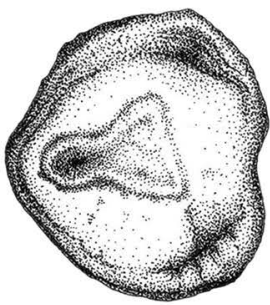

B2

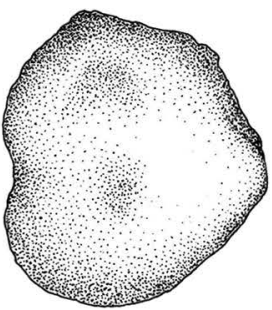

B3

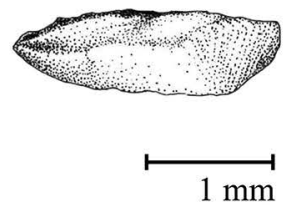

A1

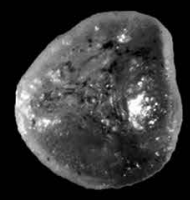

A2

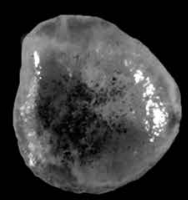

A3

\section{B1}

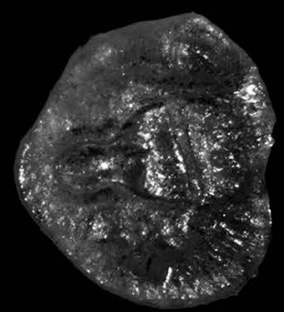

B2

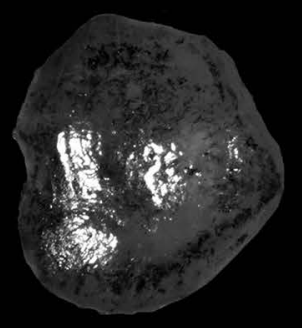

B3
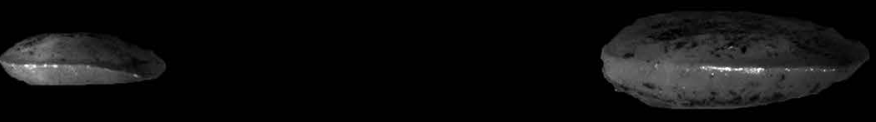

$1 \mathrm{~mm}$

$1 \mathrm{~mm}$

Plate 28. Illustrations (above) and photographs (below) of Symphurus plagusia right sagittae from fish with total length: A. $113 \mathrm{~mm}$; B. $204 \mathrm{~mm}$. The medial face is shown in A1; B1; the lateral face in A2; B2; and the ventral profile in A3; B3 (Illustration: Alexandre Arackawa; Photos: Cesar Santificetur). 